\title{
Monte Carlo simulations of densely-packed athermal polymers in the bulk and under confinement
}

\author{
Katerina Foteinopoulou, Nikos Ch. Karayiannis, Manuel Laso
}

\section{H I G H L I G H T S}

- Identification of the maximally random jammed state for polymer packings.

- Molecular simulation of the phase transition in hard-sphere chains.

- Molecular modelling of the effect of confinement in densely-packed athermal polymers.

- Numerical calculation of the topological constraints in polymeric systems.

- First-principles calculation of the scaling regimes in flexible polymers.

\begin{abstract}
A B S T R A C T
We review the main results from extensive Monte Carlo (MC) simulations on athermal polymer packings in the bulk and under confinement. By employing the simplest possible model of excluded volume, macromolecules are represented as freely-jointed chains of hard spheres of uniform size. Simulations are carried out in a wide concentration range: from very dilute up to very high volume fractions, reaching the maximally random jammed (MRJ) state. We study how factors like chain length, volume fraction and flexibility of bond lengths affect the structure, shape and size of polymers, their packing efficiency and their phase behaviour (disorder-order transition). In addition, we observe how these properties are affected by confinement realized by flat, impenetrable walls in one dimension. Finally, by mapping the parent polymer chains to primitive paths through direct geometrical algorithms, we analyse the characteristics of the entanglement network as a function of packing density.
\end{abstract}

\section{Introduction}

How particles or molecules arrange and assemble at the atomic level, under various conditions, is intimately related to the macroscopic properties and behaviour of the corresponding materials. It is thus very important to develop and employ methods and tools to analyse atomic and particulate packing in a wide range of physical systems including, but not limited to, polymers, colloids, semiconductors, composites, liquid crystals and metallic glasses. Through this a reliable structure-property relation can be established leading to novel materials with tailored characteristics. Such a scientific challenge constitutes an ideal application field for molecular simulation, a modelling field which has experienced an enormous growth since its birth more than half a century ago. Molecular modelling relies heavily on computers, whose power-to-cost ratio keeps increasing unabatedly. Furthermore, technological advances related to computer hardware are expected to continue their growth for the years to come. In parallel, scientific breakthroughs have been achieved in computational algorithms leading to very efficient numerical and simulation methods allowing the simulation of a diverse class of complex systems. Notwithstanding the obvious shortcomings related to their relatively infant state, computer simulations can robustly address "what if" questions and effectively model extreme processing conditions in an environmentally friendly, non-destructive manner. Thus, it is not surprising that in the last decades molecular simulations play a prominent role in fields as diverse as physics, chemistry, mathematics, materials science, biology and engineering.

In the present contribution we summarize the main results from extensive Monte Carlo (MC) simulations on chain packings of freely-jointed chains of hard spheres of uniform size. The 
simulated range of packing densities covers from dilute polymer packings up to jammed ones. The employed model is the simplest one for the description of polymer systems, bare of chemical details, but still incorporating excluded volume interactions which play a fundamental role in polymer physics (deGennes, 1980; Flory, 1989). The main motivation is to study how the constraints imposed by chain connectivity affect the structure, packing efficiency and phase behaviour of athermal polymer chains with respect to monomeric counterparts.

Starting from the seminal works of Bernal and co-workers in the late 1950s, a wealth of knowledge has been obtained over the years on the structural evolution of random sphere packings with increasing volume fraction $\varphi$ (Bernal, 1959, 1960; Bernal and Finney, 1967; Bernal and Mason, 1960; Scott et al., 1962). It is now widely accepted that the densest possible random packings in a 3D space, generated through different experimental setups and/ or modelling protocols, are characterized by densities in the vicinity of $\varphi^{\mathrm{MRJ}} \simeq 0.64$, subject to different generation protocols and conditions (Anikeenko and Medvedev, 2007; Anikeenko et al., 2008; Aste, 2005; Aste et al., 2005; Finney, 1970; Jullien et al., 1996; Scott and Kilgour, 1969; Tian et al., 2014). The established value is about $13.5 \%$ lower than the corresponding one for regular packings of non-overlapping hard spheres $\left(\varphi^{\text {reg }} \simeq 0.7404\right)$. The later value, initially conjectured by Kepler and proven recently by Hales (Hales, 2005; Hales et al., 2010), corresponds to the packing density of the face-centered cubic (fcc) lattice. While no such proof exists for the corresponding densest limit of random sphere packings the concept of the maximally random jammed (MRJ) state provides a precise mathematical and geometrical definition of the aforementioned state (Donev et al., 2005a, 2005b; Torquato et al., 2000).

Due to its great importance in many fields the unique characteristics of the jammed state have been studied extensively over the last years (Ballesta et al., 2008; Corwin et al., 2005; Donev et al., 2004a, 2004b, 2005a; Goodrich et al., 2012; Keys et al., 2007; Lu et al., 2008; O'Hern et al., 2002, 2003; Zexin et al., 2009). While initial studies focused understandably on dense packing of uniform spheres research has since been extended to objects with complex, anisotropic shapes (Donev et al., 2006; Frenkel et al., 1988; Haji-Akbari et al., 2013, 2011a, 2011b, 2009; Jiao et al., 2009; Jiao and Torquato, 2011; Torquato and Jiao, 2009; Veerman and Frenkel, 1991; Xu et al., 2005; Zeravcic et al., 2009). Athermal polymer packings (of hard-sphere chains) fall in the latter category: while the constituent monomers are well-defined, nonoverlapping spheres (of uniform size), the global shape and size of each molecule are highly non-trivial, fluctuate over time, and are distinctly different from one chain to the other.

In the simplest polymer representation, that of the freelyjointed chain model, connectivity is solely imposed by bonds restricting the allowed distance between successive monomers along the chain backbone; bending and torsion angles for triplets (1-3 interactions) and quartets of monomers (1-4 interactions), respectively, can fluctuate freely, limited only by the condition of excluded volume. The non-overlapping condition further dictates all non-bonded interactions between pairs. An apparent fundamental question thus arises on how polymer connectivity affects the packing efficiency, phase behaviour and jammed state compared to monomeric hard spheres. Additionally, chain connectivity endows macromolecular systems with exceptional dynamical, rheological and mechanical properties. This unique behaviour is intimately related to the uncrossability of chains which effectively leads to topological constraints between chains in the form of entanglements (deGennes, 1980; Doi and Edwards, 1988). The original reptation theory, based on the tube model, and more recent theoretical concepts have captured qualitatively and quantitatively the effect of entanglements on polymer dynamics and rheology (deGennes, 1980; Doi and Edwards, 1988; Kroger, 2004; Likhtman, 2005; Likhtman and McLeish, 2002; Marrucci, 1996; Marrucci and lanniruberto, 2004; McLeish and Larson, 1998; Ramirez et al., 2007; Stephanou et al., 2011; Wang et al., 2012). Additionally, density is one of the key factors which affect profoundly the static, dynamic and rheological properties of entangled polymers (Daoud et al., 1975; Doi and Edwards, 1988; Edwards, 1966; Fleer et al., 1993).

Molecular simulations, based on the simple athermal model, can be employed to study how packing density (volume fraction) affects not only local and global polymer structure but also the underlying entanglement network. Primarily, by exploring the whole concentration range from infinite dilution up to the MRJ state a robust definition can be provided to quantify "dense" in the commonly used term of "dense polymer". Once the jammed state is reached it is equally important to study the dependence of various quantities (for example chain size/shape, entanglement spacing, knot complexity, pair distribution function, contact network) on packing density. Such analysis will further allow the identification of the distinct scaling regimes, of the corresponding characteristic exponents and of the cross-over concentration thresholds.

Besides the physical significance in addressing the aforementioned fundamental topics there is an additional practical aspect in simulating athermal polymer packings. During the last years momentous progress has been made in the synthesis and characterization of colloidal and granular polymers (Brown et al., 2012; Palberg et al., 2009; Vutukuri et al., 2012; Zou et al., 2009). Colloidal macromolecules, by sharing key features with their atomic analogues, allow the direct experimental observation of corresponding quantities at a macroscopic level. Thus, experimental advances in colloidal polymers can greatly benefit from insights gained from detailed simulations on dense athermal polymers. Concepts like jamming (and the MRJ state), observed in computergenerated athermal polymers or in synthesized granular chains, can be related with the glass formation in polymers leading to a unified description and to an improved understanding of the phenomenon.

The phase behaviour of monomeric hard-sphere packings has been extensively studied since the earliest Molecular Dynamics (MD) simulations (Alder and Wainwright, 1957). While originally met with great scepticism and criticism, the pioneering work of Alder and Wainwright unmistakably showed, for the first time, that under specific conditions hard-sphere packings crystallize spontaneously. It is now well established that once a critical density is met and given enough observation time an initially amorphous packing of hard spheres crystallizes (Rintoul and Torquato, 1996). The entropic origins of phase transition date back to the classical work of Onsager (Onsager, 1949) on the isotropicnematic transition of hard rods and have been analysed in detail over the last years for athermal systems (Eldridge et al., 1993; Frenkel, 1988, 1999, 2000; Stroobants et al., 1987). It is now wellestablished that the face-centered cubic (fcc) is marginally thermodynamically more stable than the hexagonal close packed (hcp) crystal structure (Bolhuis et al., 1997; Woodcock, 1997). In spite of this, different ordered morphologies can also be observed in experiments and simulations like the random hexagonal close packed (rhcp) layered structure or close packed crystallites, randomly oriented with defects being strongly correlated with twinning planes (Anikeenko et al., 2007; Auer and Frenkel, 2001; Bagley, 1970; Bolhuis et al., 1997; Cheng et al., 2002; Frenkel, 1999; Harland and van Megen, 1997; He et al., 1997; Henderson and van Megen, 1998; Karayiannis et al., 2011, 2012; Kawasaki and Tanaka, 2010; Leocmach and Tanaka, 2012; O'Malley and Snook, 2003; Pusey and Vanmegen, 1986; Pusey et al., 1989, 2009; Rintoul and Torquato, 1996; Russo and Tanaka, 2012; Schilling et al., 2010; 
Zaccarelli et al., 2009). These later crystal structures can be viewed, according to Ostwald's rule (Ostwald, 1897), as intermediate (metastable) thermodynamic stages between the amorphous (random) state and the fcc crystal.

From the polymer perspective an obvious extension to the aforementioned findings is to study the effect of chain connectivity on athermal crystallization, trying to provide answers to the following questions: (i) could bond constraints frustrate, prolong or even halt crystal nucleation and growth?, (ii) if crystallization is possible, which are the similarities and differences of the established polymer crystals with respect to the monomeric ones?, and (iii) how do the crystal morphologies of freely-jointed chains of tangent hard spheres compare to the commonly-encountered lamellar structures of chemically realistic macromolecules?

The hard-sphere model is an admittedly simplified and idealized description for atoms and molecules. In spite of that, addressing the questions raised above through molecular simulations remains a highly nontrivial challenge for dense athermal polymer packings. First, there is a wide range of characteristic time and length scales that dictate the static and dynamic properties of polymers. Second, for long and highly entangled chains, topological constraints render molecular motion very sluggish; chains can only move (reptate) within the bounds of a confining tube even at intermediate concentrations. At higher densities, above the freezing transition, very slow chain dynamics, related to solidification, further render the application of brute-force MD and of conventional simulations totally impractical. Thus, a robust method must be developed and employed which would allow the efficient sampling of representative polymer configurations in the whole range of packing densities from low concentration up to the jammed state.

During the last decade Monte Carlo (MC) simulations (Allen and Tildesley, 1987; Frenkel and Smit, 2002) have proved to be an excellent alternative for the rapid equilibration of polymer melts and solutions (Theodorou, D.N., Kotelyanskii, M. (Eds.), 2007. Simulation Methods for Polymers. Dekker; Binder, K., 1995. Monte Carlo and Molecular Dynamics Simulations in Polymer Science. Oxford University Press). The main factor for the success of MC in polymer modelling is the development, over the years, of highly sophisticated algorithms which are able to sample very efficiently the phase space of polymeric systems at varied levels of chemical detail. For example in atomistic simulations such localized moves include, among others, the configurational bias (CB) (de Pablo et al., 1992a, 1992b; Laso et al., 1992; Siepmann and Frenkel, 1992) and the concerted rotation (ConRot) (Dodd et al., 1993) algorithms. Full-scale, robust equilibration even for very long chains is ensured through the application of advanced, chain-connectivity-altering moves: the end-bridging (EB) (Mavrantzas et al., 1999; Pant and Theodorou, 1995) and the double-bridging (DB) (Karayiannis et al., 2002a, 2002b). These proceed by removing and re-constructing a properly selected trimer (EB) or pair of trimers (DB) between two different chains producing two new chains whose contours are distinctly different from the original ones. EB and DB have been proven very successful in the simulation of atomistic polymers, with the full-scale of polyethylene-like (PE) systems with chains of up to thousands of monomers being achieved in modest computational time, far exceeding any alternative method (Karayiannis et al., 2002b; Mavrantzas et al., 1999). Such chain-connectivityaltering moves can be further performed in parallel pattern allowing the simulation of well-entangled, industrially-relevant polymer systems (Uhlherr et al., 2002).

While MC simulations, due to their stochastic nature, do not provide information on the dynamics of the simulated system, this is not necessarily a disadvantage. A MC scheme built around "clever" (and unphysical) MC moves is not related to the very slow ("real") dynamics of well-entangled polymers, especially at high volume fractions. Thus, a properly-devised MC scheme can provide "unphysical" equilibration of the system at rates which are by orders of magnitude faster than the "real" equilibration, as tracked by conventional, brute-force MD. Perhaps the main drawback of the MC method is that by altering the molecular detail, the chemical constitution, or the simulation conditions the efficiency of established moves is not a priori guaranteed. Consequently, major modifications or even an alternative conceptual approach may be required depending on various factors like for example the explicit representation of the hydrogen atoms in MC simulations of poly(ethylene) in atomic detail (Laso et al., 2006). The trend described above is manifestly valid not only for chemically realistic systems but also for simplified ones at very high concentrations. For example in the simulation of dense athermal packings existing MC algorithms, when directly applied to such systems, show very poor performance with increasing volume fraction. Thus, it is not surprising that past simulations on athermal polymers were limited to relatively low packing densities, below the melting transition $\varphi^{\mathrm{M}} \simeq 0.545$ (Escobedo and de Pablo, 1995; Haslam et al., 1999; Kroger, 1999; Kroger et al., 2003; Malanoski and Monson, 1997; McBride et al., 2001; Vega et al., 2001; Yethiraj and Dickman, 1992; Yethiraj and Hall, 1992).

By properly modifying existing moves for atomistic systems, we have recently proposed a MC scheme which is able to efficiently sample freely-jointed chains of hard spheres even up to the MRJ state (Karayiannis and Laso, 2008a, 2008b). In the following we will give a description of the method and will summarize the main results obtained from such MC simulations on the effect of packing density, chain length and wall confinement on structure, local packing, phase behaviour of athermal polymers, as well as on the underlying primitive path (PP) network of entanglements.

\section{Simulation method}

In $\mathrm{MC}$ simulations of athermal polymer packings the first obvious problem that one encounters is that, at high volume fractions, the acceptance rate of all local moves (reptation, rotation, internal libration (flip), etc.) becomes very small due to very frequent overlaps of the displaced monomer(s) with all unmoved ones. As the acceptance rate of the regular moves drops with increasing concentration so does the equilibration/sampling efficiency of the MC scheme. In parallel, in their original atomistic formulation the chain-connectivity-altering moves proceed by removing and adding a trimer (EB) (Pant and Theodorou, 1995) or a pair of trimers (DB) (Karayiannis et al., 2002b). As expected, the already low acceptance rate in the dilute and semi-dilute regimes diminishes at packing fractions higher than the freezing transition.

In an effort to bypass both problems we have introduced a new MC protocol tailored to simulate athermal packings even at very high volume fractions (Karayiannis and Laso, 2008a, 2008b). The two main features are that: (i) all localized moves are executed in a configurational bias pattern and (ii) $\mathrm{EB}$ (and $\mathrm{DB}$, as well as the intramolecular variants) are suitably modified so as not to entail any monomer displacement. According to (i) in a local move, instead of picking a single new (trial) position of the monomer to be displaced, $n_{\text {dis }}$ candidate positions are picked, each one corresponding to a different (randomly-selected) torsion angle. $n_{\text {dis }}$ can be selected in trial-and-error simulations by balancing and finetuning the increase in the acceptance rate and the increase in the required computational time per move. The conventional MC algorithm is recovered in the limit of $n_{\mathrm{dis}}=1$; at high volume fractions near the MRJ state, the number of trial positions is on the order of 50-80, increasing the acceptance rate of specific moves by more than one order of magnitude (Karayiannis and Laso, 2008b). 
The sampling bias introduced by the selection of $n_{\text {dis }}$ from the old state to the new one is removed by performing the reverse move in an analogous pattern and by calculating the corresponding weights of the forward (old $\rightarrow$ new) and reverse (new $\rightarrow$ old) transitions.

$E B$, by construction, introduces polydispersity in the chain length distribution. The latter is effectively controlled by casting the simulation in the semi-grand canonical statistical ensemble through proper selection of chemical potentials (Pant and Theodorou, 1995). The simplified version of EB (sEB) is executed as follows: the move is initiated when at least one pair of tangent
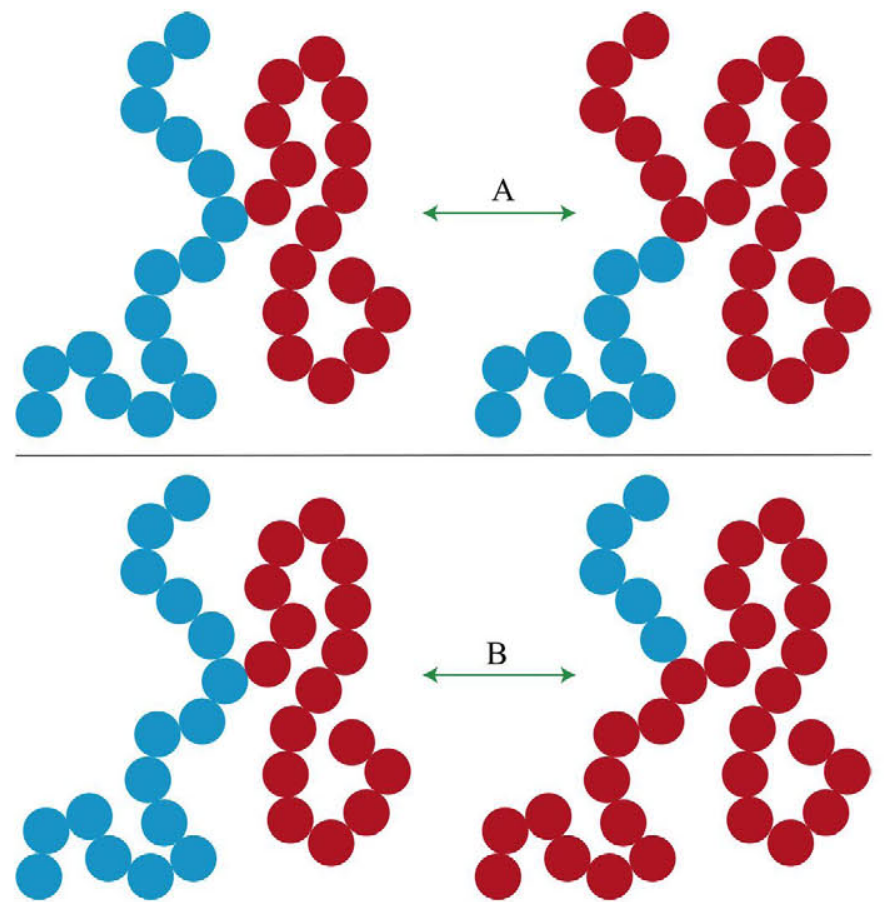

Fig. 1. Schematic representation of the simplified end-briding ( $\mathrm{sEB}$ ) move showing the two possible execution patterns in simulations of athermal polymers. Pattern applicability is subject to the requirement that the lengths of the new chains lying in the allowed interval of molecular lengths. For the move to be initiated an endmer of the red (dark grey) chain should be tangent to an internal mer of the blue (light grey) chain. (For interpretation of the references to colour in this figure legend, the reader is referred to the web version of this article.) inter-chain neighbours exists in the simulation cell, with the additional requirement than one of the two monomers is a chain end. The move proceeds by properly removing a bond and creating a new one as seen in the schematics of Fig. 1 (Karayiannis and Laso, 2008b). The bond to-be-cut (in the old state) and the bond to-be-formed (in the new state), if such pair exists, are selected in such a way that the two new chains have molecular lengths that fall within the allowed range of chain lengths. By eliminating the trimer bridge construction of the original atomistic EB algorithm and replacing it with the deletion/insertion of a single bond the performance of SEB remains very high even near the jammed state. In sharp contrast to any conventional MC moves, the acceptance rate of the SEB algorithm and thus its efficiency increases with increasing packing density (Karayiannis and Kroger, 2009; Karayiannis and Laso, 2008b). Thus, its inclusion (and that of the intramolecular variant, sIEB) in the MC scheme is imperative for the efficient sampling of the long-range chain characteristic at high volume fractions above the melting transition. Another important point to notice is that as density increases, and especially near the MRJ state, the contact network of chains (as quantified for example by the average kissing number) becomes richer (Karayiannis et al., 2009a). Thus, the critical triggering condition for the execution of sEB of finding at least one proper pair of kissing inter-chain neighbours is fulfilled much more easily and more frequently than in dilute packings. This in turn reduces the shuttling effect that often plagues MC according to which two moves of the same kind get sequentially accepted, one being the reverse of the other. Practically, because of the transitional shuttling and the mutual cancelation of the moves, the system remains in the original state; accordingly there is no impact in the equilibration even if both moves get accepted.

Simulated system sizes range from a total of 1200 up to 54,000 monomers and from average lengths of $N=12$ up to 1000 . In the original version of the MC protocol the tangency condition between bonded spheres was imposed with a fixed tolerance down to machine accuracy ( $l=\sigma$, with $l$ being the bond length and $\sigma$ the sphere diameter). In the newest version of the MC code bonds are further allowed to fluctuate in the interval $\left[l_{\min }=\sigma, \quad l_{\max }=\sigma+d l\right]$ so as to allow the investigation of the role of bond length fluctuations in the phase behaviour. This in turn allows identifying the monomer-like to polymer-like transition of the packing system as a function of bond stiffness (more details follow in Section 3.5).
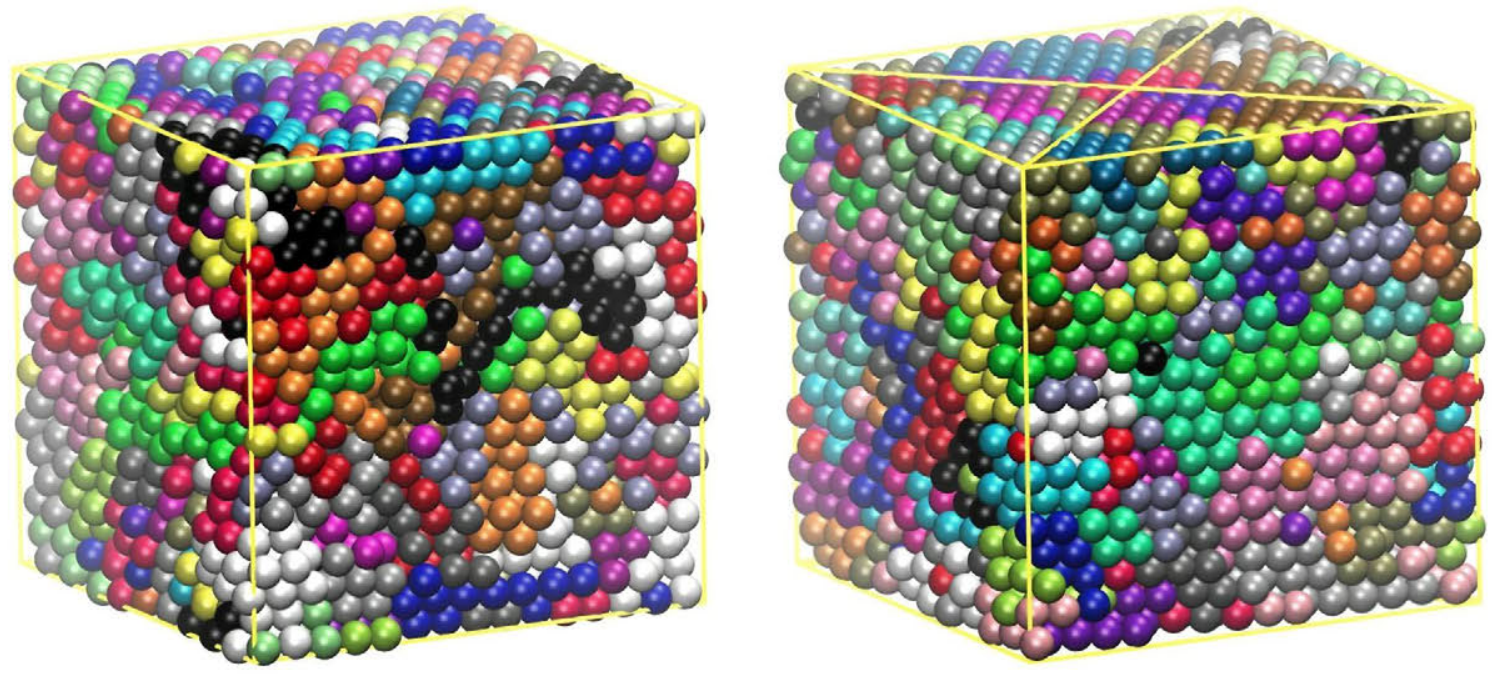

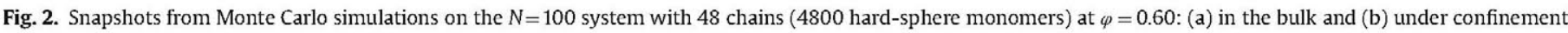

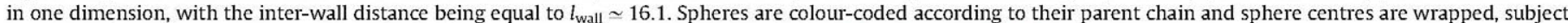
to periodic boundary conditions (where applicable). Image crated with the VMD software (Humphrey et al., 1996). 
Simulation cells are rectangular parallelepipeds; for each dimension there is a choice between periodic boundary conditions and flat, impenetrable parallel walls (plates). The former correspond to the bulk case, in the absence of interfacial and/or surface interactions. The later can be employed to study the effect of (weak or strong) confinement in one or more dimensions. Initial configurations, fully compatible with the applied simulation conditions (periodicity, confinement, bond length fluctuations, chain length distribution etc.), are generated at sufficiently low packing densities so as to guarantee the total absence of hard-sphere overlaps. Then, regular MC simulations are conducted, with volume compressions being attempted at regular intervals. Once the target density is reached, production simulations are carried out under constant volume, typically over tens or even hundreds of billions $\left(10^{9}\right)$ of $\mathrm{MC}$ steps. Typical system snapshots of dense athermal polymers in the bulk and under confinement can be seen in Figs. 2 and 3 .

In the following sections we present and analyse the main results from such extensive MC simulations with varying packing density, chain length, bond length softness and degree of confinement.

\section{Results}

\subsection{The maximally random jammed state of athermal polymers}

Jamming, and the corresponding maximally random jammed (MRJ) state in monomeric systems is intimately related to the inability of particles or atoms to move ("rattle") in the close vicinity of their local environment (Torquato et al., 2000). In a quantitative description, a particle is denoted as "rattler" if it is able to perform finite displacements without incurring overlaps with all other particles in the system which are held fixed in space. As the direct antithesis, a jammed particle is one that is effectively immobilized by the surrounding particles. Extending the concept, local jamming is related to a set of contacting particles being jammed, while global jamming demands that all particles and the corresponding contacting groups are collectively jammed (Torquato et al., 2000). Out of all jammed configurations, the MRJ state is the least ordered (or equivalently the most disordered) one (Donev et al., 2005a). Thus, to identify the corresponding MRJ state for athermal polymers, assuming that such state exists, we need to (i) analyse the degree of (dis)order and (ii) quantitatively identify jamming in chain packings.

The analysis of local structure in atomic and particulate systems can be accomplished through proper metrics including the pair radial distribution function, $g(r)$, the Voronoi tessellation, the rotationally invariant measures (Steinhardt et al., 1983) and the common neighbour analysis (Honeycutt and Andersen, 1987). Recently, we introduced a new descriptor, the Characteristic Crystallographic Element (CCE) norm to identify simultaneously radial and orientational deviations from a specific, perfect crystal structure (Karayiannis et al., 2009b; Karayiannis and Laso, 2008a). Given a reference atom and its closest neighbours (which can be identified through the Voronoi tessellation), the CCE norm is defined in such a way so as to quantify the extent to which this local structure resembles the environment of the site in a perfectly ordered structure. This is accomplished since each crystal structure is fully identified by a unique set of crystallographic operations (rotations, inversions, roto-inversions, mirror planes, etc.) (Borchard-Ott, 2002; Giacovazzo et al., 2005). In turn, each crystallographic element possesses a number of distinct elements of the symmetry groups. In general, the higher the value of the CCE norm, $\varepsilon^{X}$, the larger the discrepancy from the specific crystal structure $X$. In the case of a perfectly-ordered local environment with respect to reference $X$, the value of the corresponding CCE norm is zero $\left(\varepsilon^{X}=0\right)$. In parallel, a local environment with low CCE norm $\left(\varepsilon^{X} \rightarrow 0\right)$ with respect to crystal structure $X$ (in other words with high similarity to $X$ ) has, by construction of the metric, high CCE norm with respect to a different crystal structure $Y\left(\varepsilon^{Y} \gg 0\right)$. Consequently, the proposed metric is highly discriminating between different crystal structures. This feature renders the CCE norm a robust descriptor to accurately identify crystal nucleation and growth in general atomic and particulate systems (Hoy and Karayiannis, 2013; Karayiannis et al., 2009b, 2009c,2011, 2012, 2013a, 2013b, ; Wu et al., 2014). The mathematical formulation of the CCE descriptor along with details on the algorithmic implementation can be found elsewhere (Karayiannis et al., 2010; Karayiannis et al., 2009b, 2013b). As reported in Section 1, for dense hard-sphere packings the emerging and prevailing crystal morphologies consist of close packed sites. Thus, for athermal polymers local structure is identified through the calculation of the fcc, hcp and fivefold CCE norms (Karayiannis et al., 2011, 2012).
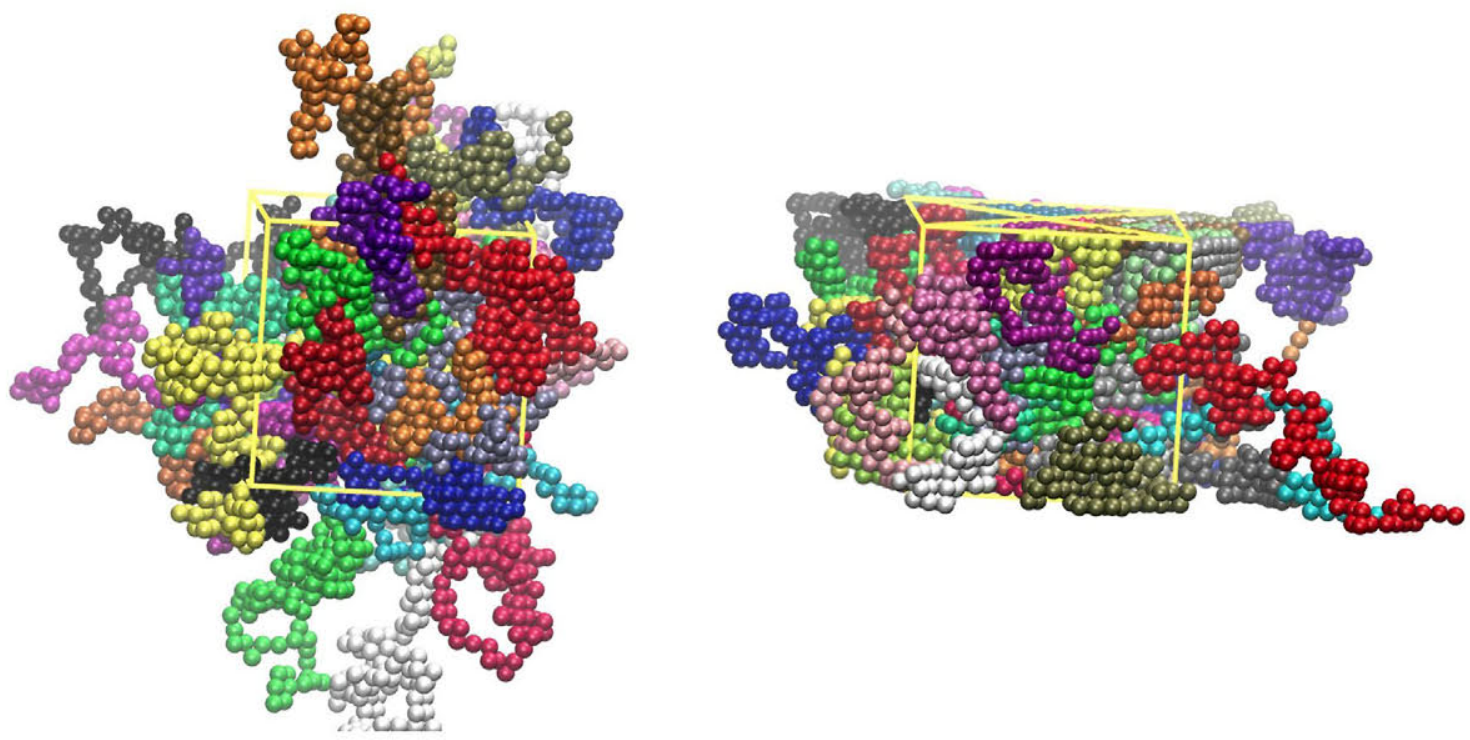

Fig. 3. Same as in Fig. 2, but with coordinates of sphere centres fully unwrapped in space. Image crated with the VMD software (Humphrey et al., 1996). 
The CCE norm is thus the structural descriptor used to measure the degree of (dis)order in the generated hard-sphere chains. In pursue of the MRJ state of polymer packings the next obvious task is to quantify jamming. Due to the constraints imposed by chain connectivity, direct application of the "rattler" concept is not possible in chain systems. However, a similar scheme can be devised where each hard-sphere monomer attempts minute displacements taking into account the imposed bond constraints. For spheres which are either internal monomers or chain ends, such displacements take the form of flip- or rotation-like moves, respectively. If all attempted moves lead to overlaps with all other monomers, which are held fixed in space, the particle is effectively jammed.

The degree of ordering (crystallinity) and the fraction of jammed monomers are continuously measured throughout the MC simulations. With increasing packing density, the number of sites with hcp or fcc similarity increases but remains at very low levels compared to the bulk of the system. In parallel, no appreciable increase is observed for the fraction of jammed sites even at high volume fractions. However, subject to statistical fluctuations, based on the generation protocol and the stochastic nature of the process, once a critical packing density is met the population of "flippers" decreases sharply. At $\varphi^{\mathrm{MRJ}} \rightarrow 0.64$ jammed sites constitute the overwhelming majority of monomers. The precipitous increase of jammed spheres combined with the very low degree of ordering (quantified by the CCE norm) are the two definite traces to identify the proximity to the MRJ state in athermal polymer packings as seen in Fig. 4.

Based on the above it can be concluded that freely-jointed chains of tangent hard spheres can be packed as efficiently and as densely as their monomeric analogues, reaching equivalent MRJ states, $\varphi^{\mathrm{MRJ}}$ (chains) $=\varphi^{\mathrm{MRJ}}$ (monomers) (Karayiannis et al., 2009d, 2013a; Karayiannis and Laso, 2008a; Laso et al., 2009). This is a non-trivial finding given that chain connectivity endows polymer packings with constraints which are absent in monomeric assemblies. If conclusive proof existed (and not just numerical evidence) for the correctness of the observed volume fraction at the MRJ for monomeric spheres, one could conclude that it would be impossible for chains to pack more densely. In the absence of such a proof, our work very strongly supports the natural conjecture that hard-sphere chains pack as densely as monomeric spheres. The unique nature of chain packings compared to monomeric analogues can be understood through simple arguments: At the local level the presence of bond constraints alter the contact network of each site. Equally important, at the global level topological

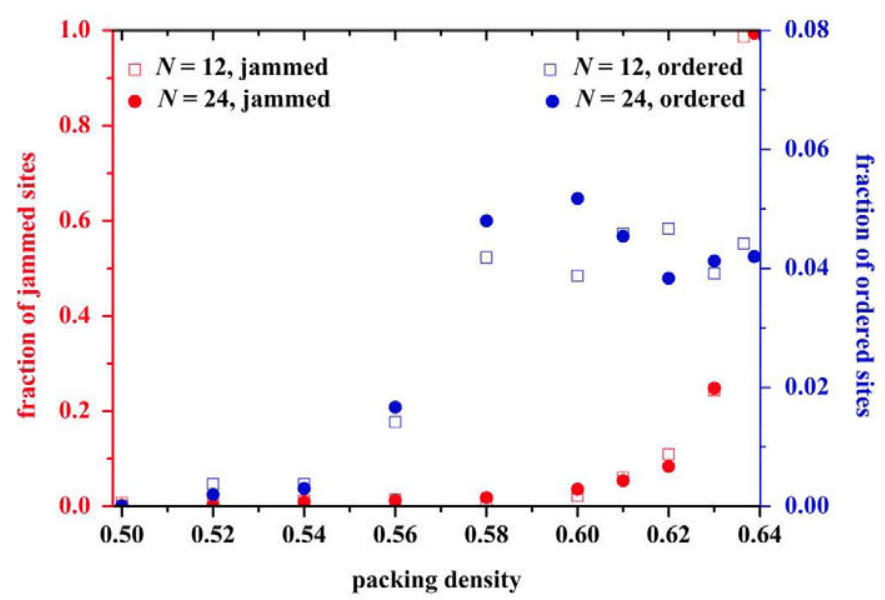

Fig. 4. (Left $y$-axis) Fraction of jammed sites and (right $y$-axis): fraction of ordered sites as a function of packing density $\varphi$ as obtained from MC simulations on the $N=12$ and 24 systems. constraints in the form of entanglements exist between chains, which are expected to significantly affect their packing ability, especially at very high densities.

The identification of the MRJ state for hard-sphere chains, primarily answers the fundamental question of the densest possible structure in random polymer packings and how this state compares to the one of monomeric analogues. Furthermore, it provides a definitive, precisely-bounded range $\left(0 \leq \varphi \leq \varphi^{\mathrm{MRJ}}\right)$ to study how structure, size/shape, phase behaviour and topological constraints (entanglements) of flexible chains evolve with packing density. In practical terms, a well-defined classification is offered to eliminate relative arbitrariness in terms and concepts related to polymer physics (for example "dense polymer", "chain collapse" etc).

\subsection{Local chain structure}

Chain connectivity imposes constraints which force bonded spheres to adopt specific local configurations which are absent in monomeric counterparts. Obviously, with respect to bond lengths the tangency condition $(d l \rightarrow 0)$ results in an inherent contact network. Each sphere-monomer, depending on the position along the chain possesses a kissing number of 2 (internal monomer) or 1 (chain end), with potential implications in the vicinity of the MRJ state (Karayiannis et al., 2009a).

Various potentials and corresponding formulas can be employed to control contributions arising from other types of bond constraints. According to the freely-jointed model adopted here, apart from the bond constraints all other terms, primarily the bending and torsion angles, are allowed to fluctuate freely. In spite of it, and given that all covalent contributions remain subjected to excluded volume interactions, packing density has a profound effect on the conformations of bonded geometry. This can be clearly seen by the evolution of the bending and torsion angle distributions with increasing volume fraction as obtained for the $N=100$ system in the bulk (Fig. 5) and under confinement (Fig. 6). At very low volume fractions (dilute system) bending and torsion angles are determined solely by intramolecular interactions imposed by bond constraints. As a result, the behaviour can be accurately captured by considering an ideal system of isolated chains with excluded volume being effective for monomers separated by up to 3 bonds (Karayiannis et al., 2009d). As packing density increases chains progressively interact with each other leading to very compact configurations especially in the vicinity of the MRJ state (Karayiannis and Laso, 2008a). Bending and torsion angles adopt specific, favoured arrangements in order to locally minimize free volume. This trend is the main factor for chain compactness, leading, once a critical density range is reached, to the phenomenon of "chain collapse" as will be explained in the following section.

As shown in Karayiannis et al. (2009d, 2013a) specific local arrangements corresponding to prominent peaks in the bending and torsion angle distributions of dense chain assemblies, can be mapped into the polytetrahedral model of disordered hard-sphere packings (Anikeenko and Medvedev, 2007; Anikeenko et al., 2008).

\subsection{Characteristic scaling regimes}

Given the pronounced effect of volume fraction on local configurations leading to characteristic local arrangements, it is not surprising that packing density further affects global chain size and shape. Scaling laws predicting the dependence of dynamic and static properties of polymer melts and solutions have been pioneered by de Gennes and coworkers (Daoud et al., 1975; deGennes, 1980; Farnoux et al., 1978). Macromolecular dimensions 

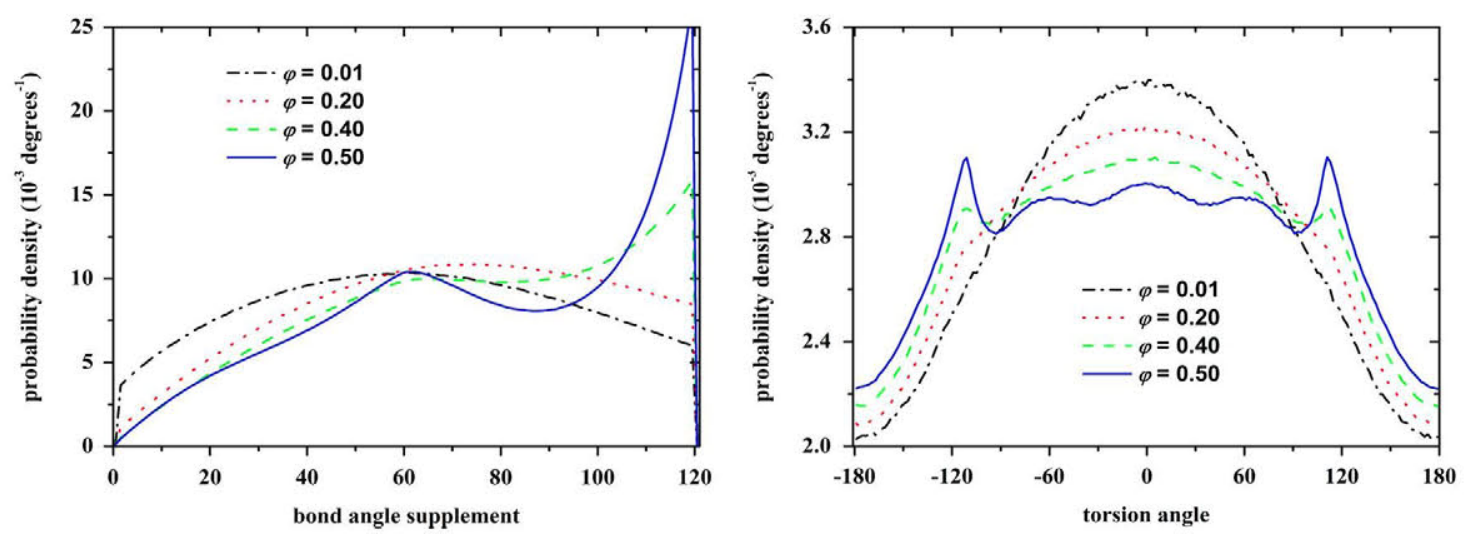

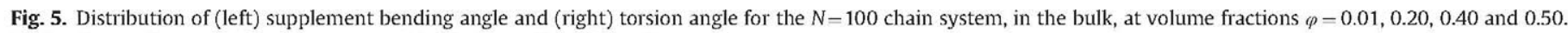
For torsion angles, zero degrees correspond to the trans $(t)$ conformation.
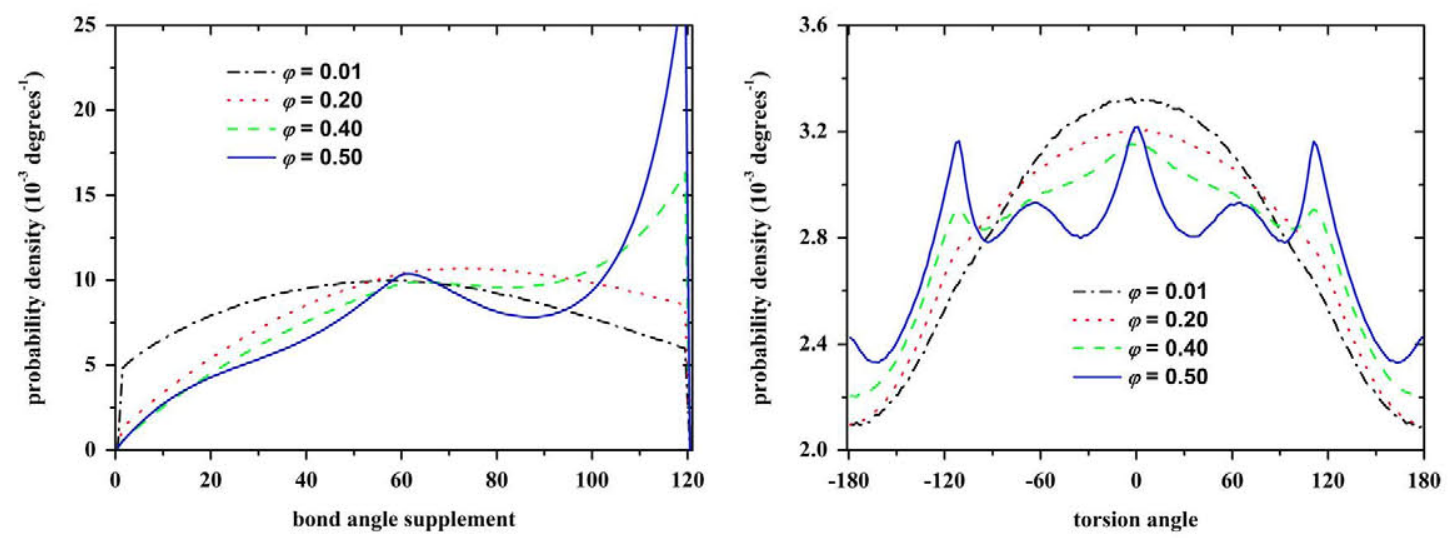

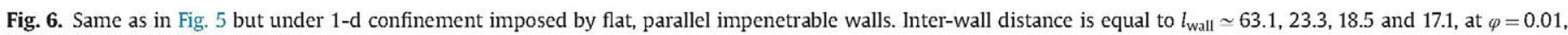
$0.20,0.40$ and 0.50 , respectively.

can be quantified through the characteristic ratio, $C_{N}$, defined as $C_{N}=\left\langle R^{2}\right\rangle /(N-1) / l^{2}$, where $\left\langle R^{2}\right\rangle$ is the mean square end-to-end distance, $(N-1)$ is the number of bonds along the chain backbone and $l$ the bond length (here equal to sphere diameter). The logarithm of $C_{N}$ as a function of the logarithm of packing density can be seen in Fig. 7 as obtained from MC simulations on the $N=50,100$ and 1000 chains of tangent hard spheres in the bulk. From these simulation data we can accurately identify the scaling regimes along with their boundaries and characteristic exponents on the dependence of chain size on volume fraction $\left(C_{N} \sim \varphi^{n}\right)$. According to simulation findings (Foteinopoulou et al., 2008; Laso and Karayiannis, 2008; Laso et al., 2009), four distinct scaling regimes can be clearly ascertained, in accordance to theoretical predictions (Fleer et al., 1993), for flexible chains: (i) dilute $\left(0 \leq \varphi \leq \varphi^{*, \text { semi }}\right): C_{N} \sim \varphi^{0}$, (ii) semi-dilute $\left(\varphi^{*, \text { semi }} \leq \varphi \leq \varphi^{*, \text { marg }}\right)$ : $C_{N} \sim \varphi^{-0.23}$, (iii) marginal $\left(\varphi^{*, \text { marg }} \leq \varphi \leq \varphi^{*, \text { conc }}\right): C_{N} \sim \varphi^{-1.00}$ and (iv) concentrated ( $\left.\varphi^{*, c o n c} \leq \varphi \leq \varphi^{\mathrm{MRJ}}\right)$ : $C_{N} \sim \varphi^{0}$. In more detail, with respect to the scaling exponent in the semi-dilute regime $\left\langle R^{2}\right\rangle \propto N \varphi^{\gamma}$ with $\gamma=(1-2 \nu) /(3 \nu-1), \nu$ being the Flory exponent $(\nu=0.588$ ) (Chaikin and Lubensky, 2000). corresponding crossover thresholds have been proposed to be $\varphi^{* \text { semi }}(N) \simeq N^{-0.764}$ (Cotton et al., 1976), $\varphi^{* \text {,marg }} \simeq \varphi^{\mathrm{MRJ}} / 2$ and $\varphi^{* \text {,conc }} \simeq 0.59$, the latter two being proposed through graph theoretical proof (Laso and Karayiannis, 2008). The predicted values for the threshold concentrations have been confirmed by present MC simulations.

The scaling regimes along with the corresponding exponents, as established here, are in excellent agreement with past theoretical predictions and available experimental data (Cotton et al., 1976; Daoud et al., 1975; deGennes, 1980; Edwards, 1966; Fleer et al.,

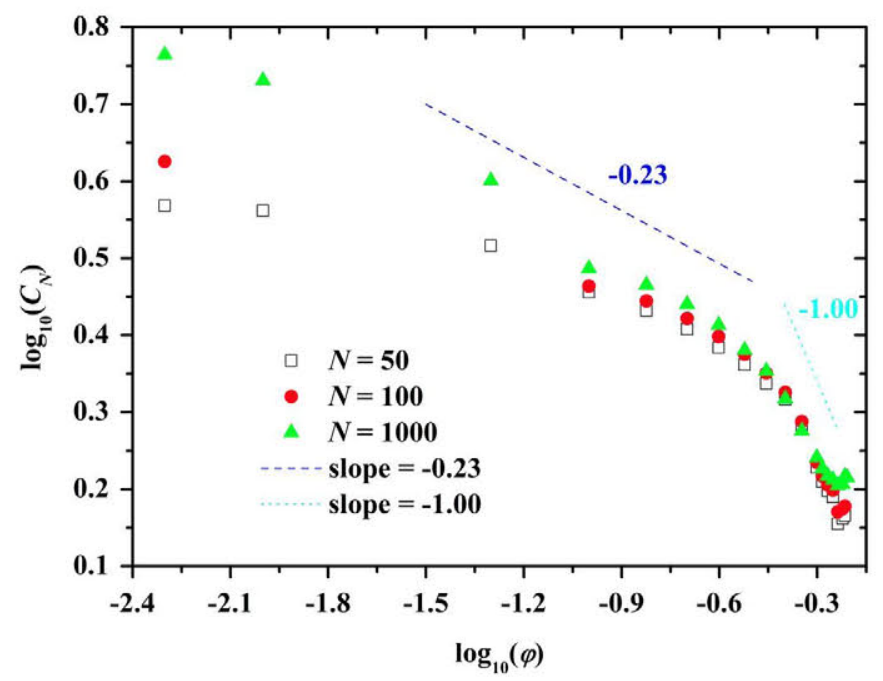

Fig. 7. Logarithm of the characteristic ratio, $\log _{10}\left(C_{N}\right)$, as a function of the logarithm of packing density, $\log _{10}(\varphi)$, as obtained from MC simulations on the $N=50,100$ and 1000 chain systems. Lines with slopes of -0.23 and -1.00 are drawn as guides for the eye.

1993; Laso and Karayiannis, 2008). By identifying the concentration ranges, one can further relate structural and conformational transitions, at both local (monomer) and global (chain) levels, with the onset of the corresponding regime. Furthermore, even if for the identification of the scaling regimes and of the characteristic 
exponents the athermal chain model was employed, the present findings are expected to be manifestly valid also for flexible polymer models with more realistic chemical detail.

\subsection{Scaling of entanglements}

The exceptional dynamic and rheological behaviour of polymer melts and solutions is attributed to chain uncrossability leading to the formation of topological constraints (entanglements). Polymer rheology is intimately related to the concept of entanglements, which lies at the heart of many macromolecular theories (deGennes, 1980; Doi and Edwards, 1988; Likhtman and McLeish, 2002). In this framework, according to the original "tube" model (deGennes, 1980) and the reptation theory (Doi and Edwards, 1988) inter-chain topological constraints restrict chain motion in a "tube-like" region. The axis of the tube, or in other words the primitive path of the chain with its contour length $L_{\mathrm{pp}}$, the tube diameter $a_{P P}$ (more precisely the Kuhn step length of the tube), and the molecular weight between entanglements $N_{\mathrm{e}}$ constitute the key elements of the tube theory. The latter is frequently used to link the theory with experimentally observed rheological properties of polymeric melts through its connection to the plateau modulus (Everaers et al., 2004; Fetters et al., 1999; Foteinopoulou et al., 2009; Theodorou and Tzoumanekas, 2006; Tzoumanekas et al., 2009). Additionally, the derived entanglement statistics can be mapped (Anogiannakis et al., 2012; Foteinopoulou et al., 2008; Steenbakkers et al., 2014) into novel slip-link theories that describe the rheological behaviour of polymeric liquids (Khaliullin and Schieber, 2009; Likhtman, 2005; Marrucci and Ianniruberto, 2004; Masubuchi et al., 2003; Ramirez et al., 2007; Stephanou et al., 2011; Wang et al., 2012).

According to the original tube theory, the primitive path (PP) of a chain is defined as the shortest path that connects the chain ends retaining the topology of the original chain without violating the topological constraints imposed on it from the other chains and whose length decreases continuously during the minimization process (Doi and Edwards, 1988). For a collection of chains the primitive path is the shortest multiply disconnected path (Doi and Edwards, 1988; Kroger, 2005).

During the last decade, a significant amount of modelling studies have been devoted to construct the PP network of a collection of polymeric chains, to identify entanglements and to compute all related quantities (Everaers et al., 2004; Kroger, 2005; Shanbhag and Larson, 2005; Tzoumanekas and Theodorou, 2006). The algorithms, used to extract the PP network from the original configurations, can be split into two main categories: they either compute the PP network in the course of an energy minimization procedure based on the original PPA algorithm of Everaers et al. (2004) or they rely on a computationally efficient geometric length minimization method like the Z1 (Kroger, 2005) and CReTA algorithms (Tzoumanekas and Theodorou, 2006). It has been established, that subject to small statistical fluctuations, both the $\mathrm{Z} 1$ and CReTA methods provide similar results with respect to the topology and average contour length of the primitive paths (Foteinopoulou et al., 2006; Tzoumanekas and Theodorou, 2006). Moreover the PPA and Z1, when applied to the same configuration of polymer chains, give similar results for the mean primitive path contour length, the error being less than 10\% (Hoy et al., 2009).

Independent analyses using the length minimization algorithms have demonstrated that PPs do not follow random walk statistics (Foteinopoulou et al., 2009; Tzoumanekas and Theodorou, 2006). The geometric algorithms further provide a direct measure of the number of kinks (entanglements) and accordingly a quantitative description of the underlying PP network. The reported relation between PP network-related quantities, invoking the random coil assumption, and corresponding ones obtained by direct measure of the PP mesh was recently interpreted through the concepts of rubber elasticity and treating entanglements as binary contacts (Everaers, 2012).

In the present study the primitive paths of athermal polymer packings are extracted through the Z1 algorithm (Karayiannis and Kroger, 2009; Kroger, 2005). Fig. 8 shows the derived PP network for the $N=1000$ chain system together with the parent chain configurations at $\varphi=0.60$. In parallel, Fig. 9 shows a snapshot focusing on an isolated, randomly picked, hard-sphere chain and the corresponding primitive path as obtained through the $\mathrm{Z} 1$ algorithm. Motivated by the corresponding scaling laws for the "parent" chain molecules one can further obtain the corresponding scaling trends also for the primitive paths (Foteinopoulou et al., 2008; Laso et al., 2009).

An alternative measure of topological constrains in macromolecules can be obtained through the concept of intra-chain knot which is gaining interest in the last years (Foteinopoulou et al., 2008; Koniaris and Muthukumar, 1991; Laso et al., 2009; Mansfield, 2007a, 2007b; Panagiotou et al., 2013; Qin and
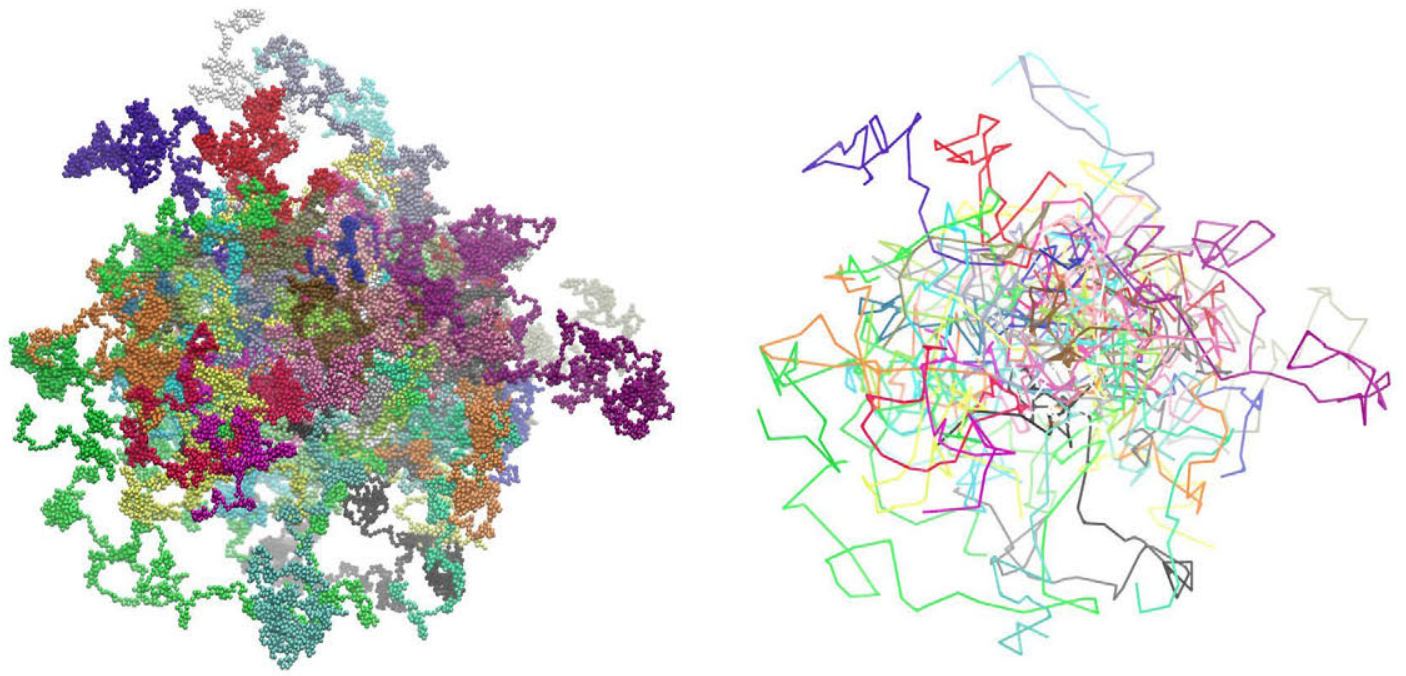

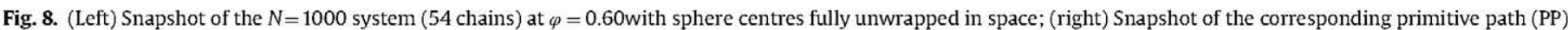

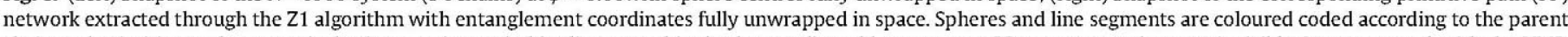

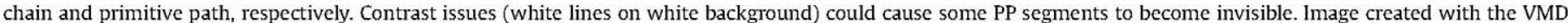
software (Humphrey et al., 1996). 


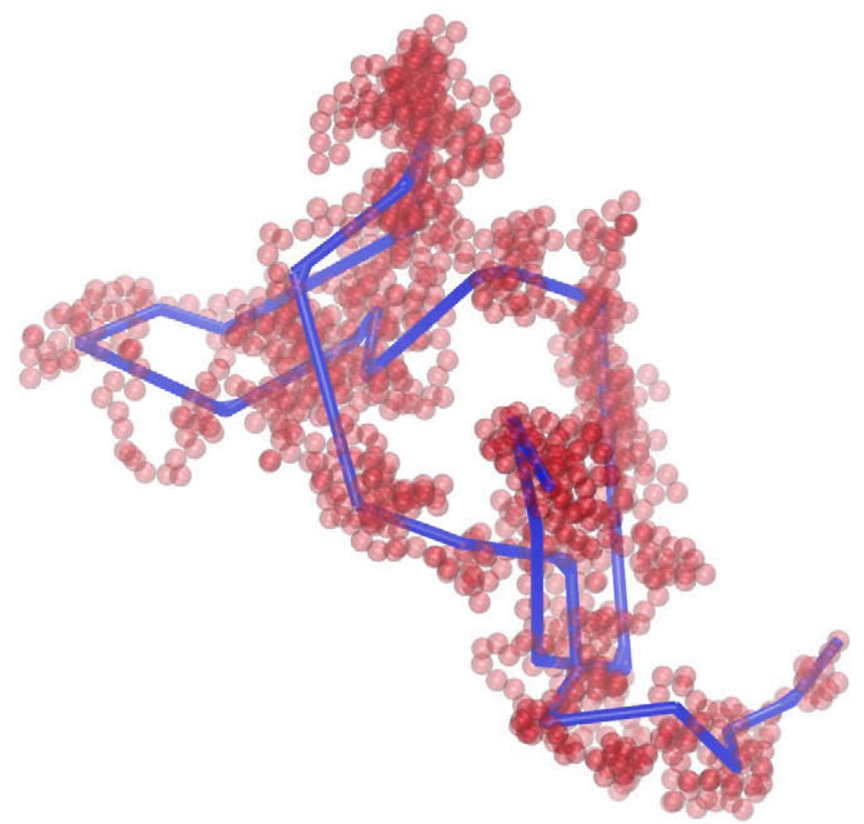

Fig. 9. Parent configuration of a single chain, randomly picked from the 54-chain $N=100$ system simulated at $\varphi=0.60$, along with the corresponding primitive path (PP). Image created with the VMD software (Humphrey et al., 1996).

Milner, 2011; Rawdon et al., 2008a, 2008b). It is thus tempting to try to correlate the two different forms of topological constraints: entanglements, of inter-chain origin, with knots, an intramolecular topological quantity. A connection between polymer knots and entanglement distance $N_{\mathrm{e}}$ was established based on theoretical arguments on the topological entropy of long entangled polymers (Qin and Milner, 2011).

Our studies reveal that for flexible chains there is a direct connection between entanglements and knots in the whole range of volume fraction (Foteinopoulou et al., 2008; Laso et al., 2009). We use the technique proposed by Mansfield (2007a, 2007b) to identify knots, which is based on the identification of the knot group instead of the knot invariants used in other studies. Starting from an arbitrary projection of an embedded graph, this method generates a sequence of representations any of which is a full and complete representation of the knot group. This sequence is compared against the entries in a previous look-up table and if a match is found the knot group is identified. Knots are defined for closed paths, therefore to apply the knot analysis the linear chains of our study are converted into closed polygons (Foteinopoulou et al., 2008; Laso et al., 2009). Numerous closure schemes can be adopted to study knots in linear chains (Mansfield, 2007a). In the present work two approaches are adopted: the most trivial one simply connects the chains ends through a straight line, the other projects rays outward through the ends until they intersect a sphere which entirely circumscribes the chain, then proceeds by connecting the two rays using a great circle path between the intersection points. It is observed that both methods provide essentially the same results on the scaling of the knot state and complexity with volume fraction (Foteinopoulou et al., 2008).

In Fig. 10 the fraction of knotted chains is compared with the number of entanglements of the PP network as a function of packing density for the $N=1000$ system. The population of entanglements is measured by two different approaches: (i) by directly counting the number of interior kinks in the primitive paths, $Z_{\text {kink }}$, and (ii) by adopting the random coil assumption for the PPs (Doi and Edwards, 1988; Everaers et al., 2004). According to the later hypothesis the number of entanglements can be

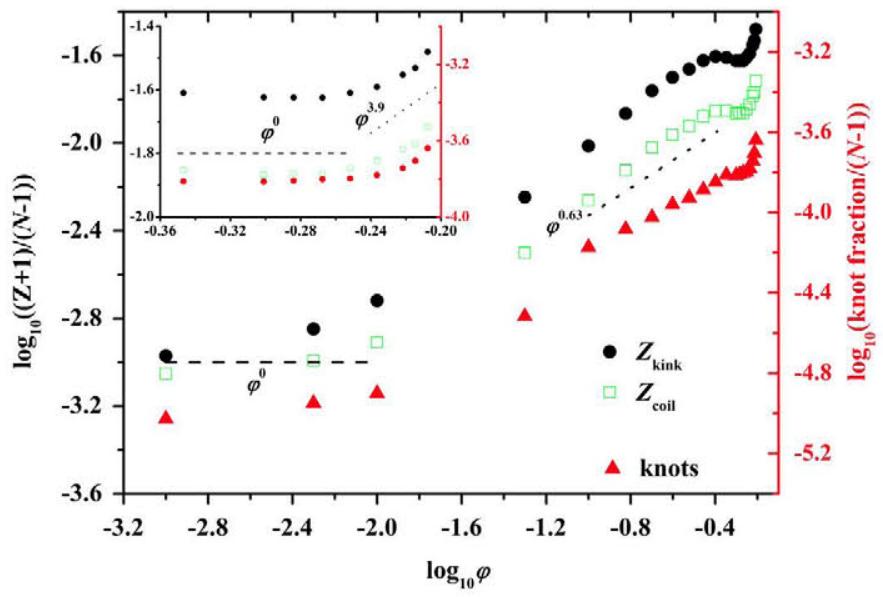

Fig. 10. (Left) logarithm of the average number of primitive path segments (divided by the number of bonds) and (right) logarithm of the fraction of knotted chains (divided by number of bonds) as functions of the logarithm of packing density. Inset: zoom in the marginal and concentrated regimes. Lines with characteristic slopes are drawn as guides for the eye.

extracted from the PP contour length $L_{\mathrm{PP}}$ and the end to end distance $R_{\text {ee }}$ as $\mathrm{Z}_{\text {coil }}=(\langle\mathrm{N}\rangle /\langle\mathrm{N}-1\rangle)\left(\left(\mathrm{L}_{P P}^{2} / \mathrm{R}_{e e}^{2}\right)-1\right)$.

Of particular interest is the observation that both entanglements and knots exhibit the same scaling regimes, further characterized by the same crossover concentrations and scaling exponents (Foteinopoulou et al., 2008). Although at first glance entanglements and knots seem uncorrelated, the multi-chain phenomenon of entanglements leaves its mark on the shape of the individual chains which in turn affects the knot state (Laso et al., 2009).

The inter-entanglement distance $N_{\mathrm{e}}$ can be trivially derived from the PP information so as to investigate its variation with volume fraction. For the calculation of the entanglement distance we adopt the simple $M_{\text {kink }}$ ideal estimator proposed in Hoy et al. (2009). Through such estimators the PP statistics in the (longchain) polymeric regime can be predicted through application to significantly smaller (short-chain) and computationally-expedient systems. The derivation of $M_{\text {kink }}$ is based on measuring directly the interior kinks in the PP instead of adopting a random coil assumption for the primitive paths; it is defined as the inverse of the slope in the linear regime of the $\langle\mathrm{Z}\rangle$-versus- $N$ curve. In order to apply this measure, chain lengths in system configurations are required to span a spectrum of different $N$ values with $N \geq N_{1}, N_{1}$ being the threshold value above which $\langle\mathrm{Z}\rangle$ becomes linear with $N$. It has been shown for atomistic and bead-spring polymer systems (Foteinopoulou et al., 2009; Hoy et al., 2009) and further verified for systems of hard-sphere chains, that $\langle\mathrm{Z}\rangle$ scales linearly with $N$ even when $\langle Z\rangle$ is less than unity. Therefore the proposed estimator is readily applicable to the majority of the simulated polydisperse athermal systems. For example the simulated $N=1000$ system contains chains that span the length interval $600 \leq N \leq 1400$. The $M_{\text {kink }}$ estimator can be derived for the long-chain $N=1000$ and be directly compared with the corresponding predictions as obtained from systems characterized by smaller average chain lengths. The latter are properly chosen so as to have a mean molecular length close to the expected $N_{\mathrm{e}}$ value (usually with $N \sim 50-100$ ). Fig. 11 shows the dependence of $N_{\mathrm{e}}$ on packing density demonstrating the ability of the $M_{\text {kink }}$ predictor to give accurate estimations on the entanglement spacing for systems with chain lengths deep in the polymeric regime by employing data from short-chain or oligomeric systems.

Detailed information can be deducted on the static properties of the PP network (entanglement statistics) given that MC simulations do not provide dynamical information. For example the cumulative probability of the PP length as obtained from MC 


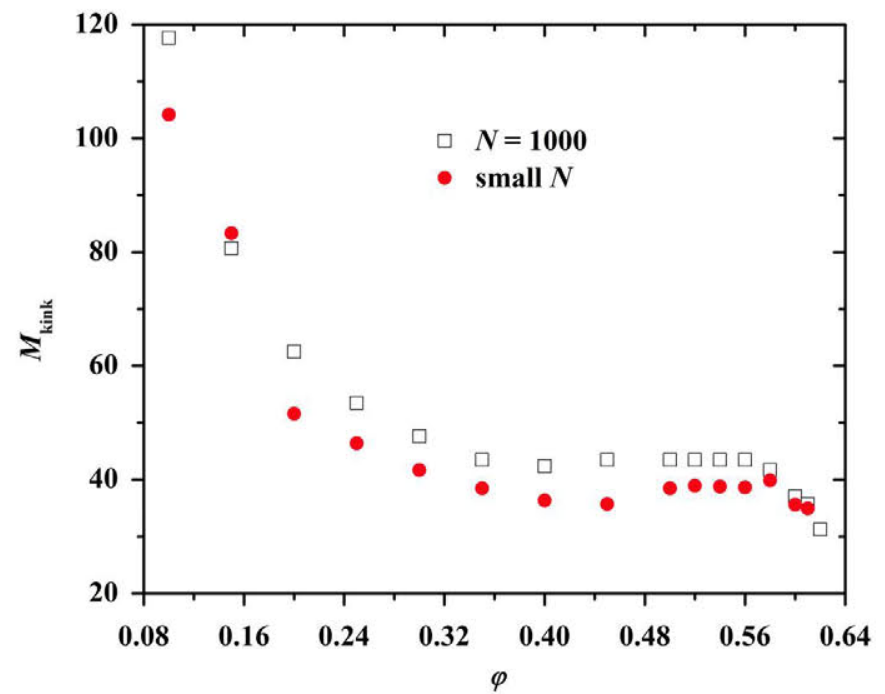

Fig. 11. Inter-entanglement distance, $M_{\text {kink }}$, as a function of packing density, $\varphi$, as obtained from the $N=1000$ polydisperse system and from systems of significantly shorter chains $(N=50-100)$.

simulations of entangled athermal chains (Foteinopoulou et al., 2008 ) is in excellent agreement with the predictions of the thermodynamically-consistent slip-link model by Khaliullin and Schieber (2009). Same is true when we compare the distribution of the entanglement spacing against the exponential-type master curve proposed by Tzoumanekas and Theodorou (Kamio et al., 2007; Tzoumanekas and Theodorou, 2006).

\subsection{Phase behaviour of hard sphere chains}

As reported in Section 1 it is well established, through experiments and simulations, that, once a critical volume fraction is met and given enough observation time, monomeric packings of uniform hard spheres transit from the initial amorphous (random) state to the final crystalline (ordered) one. An obvious question is how chain connectivity affects the phase behaviour. Furthermore, if athermal polymers crystallize how do their phase diagram and the established ordered morphologies compare with the ones of monomeric analogues?

To study the phase behaviour of hard sphere chains we extend significantly the reported MC simulations to reach hundreds of billions $\left(10^{11}\right)$ of steps. The local environment of each spheremonomer is analysed, at regular trajectory recordings, to identify the degree of similarity to the hcp and fcc crystals and the fivefold local symmetry. The evolution of the degree of ordering (crystallinity), quantified here as the sum of close packed (hcp and fcc) sites, is tracked by the application of the CCE norm descriptor as explained earlier (Karayiannis et al., 2009b). Fig. 12 shows the fraction of hcp- and fcc-like spheres versus MC steps from the $N=12$ at $\varphi=0.56$ and 0.61 . At $\varphi=0.56$ the population of ordered sites remains very low throughout the whole simulation. However, at higher concentration $(\varphi=0.61)$ a sharp increase is observed in the fraction of hcp- and fcc-like spheres as the simulation progresses. This abrupt growth marks vividly the disorder-order transition which eventually leads to the formation of well-defined crystal structures. Thus, it is evident that at adequately high volume fractions and given enough time, chains of tangent hard spheres (of uniform size) crystallize as their monomeric analogues do (Karayiannis et al., 2010, 2013a, 2013b). Similar findings, confirming crystal nucleation and growth in dense athermal polymer packings have been independently reported in $\mathrm{Ni}$ and Dijkstra (2013) from dynamic simulations on chains with soft bond lengths.

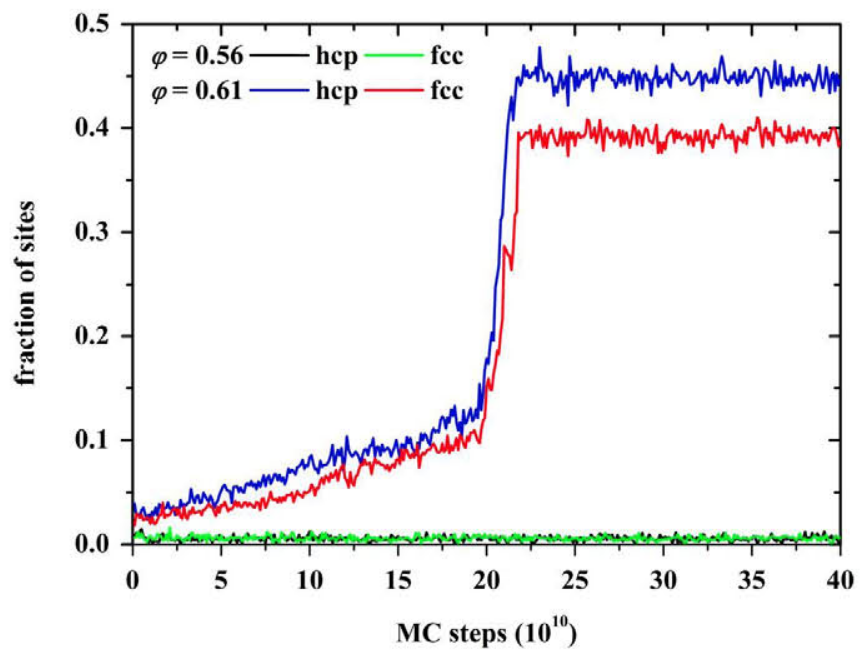

Fig. 12. Fraction of closed packed sites (with hcp and fcc similarity), as quantified by the CCE norm, versus MC steps as obtained from simulations on the $N=12$ system at $\varphi=0.56$ and 0.61 .

In an isolated, athermal polymer system, given the absence of any energetic interactions (apart from the non-overlapping condition of excluded volume), entropy is the driving force that leads the phase transition. This fundamental physical insight has been established for the first time in the classical work of Onsager (1949) on the isotropic-nematic transition of hard rods and the entropy-driven phase transitions have since been studied extensively in athermal systems (Eldridge et al., 1993; Frenkel, 1994, 1999, 2000; Frenkel et al., 1988).

As explained in detail in Karayiannis et al. (2010, 2009c), as the originally disordered packing transits to the ordered state, the local environment becomes more spherical and more symmetric. To quantify the structural transition we first identify the enclosing polyhedron around each site as determined from Voronoi tessellation. The shape of each Voronoi cell is calculated through two principal measures, asphericity $b$ and relative shape anisotropy $k^{2}$. These quantities can be calculated from the eigenvalues of the moment of inertia tensor of the Voronoi cell by treating all vertices as points of unit mass (Karayiannis et al., 2009b, 2009c, 2010, 2013b). The lower the value of $b$ the higher the resemblance of the object to a sphere and the lower the $k^{2}$ value the higher the isotropy of the shape with respect to the co-moving principal axes frame defined by the normalized eigenvectors. By tracking the evolution of asphericity and relative shape anisotropy as a function of MC steps we observe that both measures show a very sharp drop as the system transits to the ordered state. Thus, it can be concluded that the local environment around each site becomes more spherical and more symmetric in the ordered (crystal) phase (Karayiannis et al., 2009c, 2010, 2013b).

This, in turn, enhances the ability of hard-sphere monomers to move in their local vicinity. Accordingly, a significant increase is observed in the translational entropy of the system, more than compensating for the corresponding decreases in the conformational and orientational contributions in entropy (Karayiannis et al., 2010). A relation between the structural alternations of the local environment and the ability of monomers to explore them efficiently can be established through the concept of "flipper", which has been introduced earlier for the identification of the MRJ state. A "flipper" (or a "rattler") is in general a monomer which is able to perform small displacements locally. It is seen that the number of "flippers" increases significantly in the ordered phase where the local environment is more spherical and more symmetric compared to the disordered one (Karayiannis et al., 2009c, 2010). Accordingly, our results support the conjecture that the 
stable phase of dense athermal packings is a crystal consisting of hcp- and fcc-like (close packed) sites. For tangent bonded monomers, ordered morphologies consist of well-defined, stack-faulted layers (of either hcp- or fcc-like character) with a single stacking direction, a structure characteristic of the random hexagonal close packing (rhcp) (Karayiannis et al., 2010, 2009c).

While the phase-behaviour of athermal chains shares a wealth of similarities with the corresponding one exhibited by monomeric counterparts, there exist substantial differences as well. In the limit of tangent monomers $(d l \rightarrow 0)$ and at packing densities near and slightly above the melting point (of monomeric systems), chain connectivity frustrates crystallization (Karayiannis et al., $2013 \mathrm{~b}$ ). For example, at $\varphi=0.56$, freely-jointed chains of tangent hard spheres remain amorphous (Karayiannis et al., 2009c, 2010), while monomeric packings show clearly a first-order phase transition (Karayiannis et al., 2011, 2012).

It can therefore be concluded that constraints related to chain connectivity affect the phase behaviour of athermal chains near melting. To study this effect in detail we additionally allow bond lengths to fluctuate in an interval $\left[l_{\min }=\sigma, \quad l_{\max }=\sigma+d l\right]$ with varied value of $d l$ (bond softness) compared to the tangent limit $(d l \rightarrow 0)$. As the bond constraints become weaker, a wealth of ordered structures emerge, ranging from random stacks to pure hcp and fcc crystallites, at concentration ranges where the strictly tangent systems do not crystallize. Representative system snapshots in the ordered phase can be seen in Fig. 13 as obtained from extensive MC simulations on the $N=12$ system at $\varphi=0.5575$ with varied bond softness. For comparison, according to the data shown in Fig. 12 the corresponding tangent system with $d l \rightarrow 0$ shows no traces of disorder-order transition at $\varphi=0.56$.

\subsection{Athermal polymers under confinement}

MC simulations are extended to study the effect of confinement on the static and conformational properties as well as on the phase behaviour of athermal polymers. Confinement is realized through the presence of flat, parallel, impenetrable walls in (at least) one dimension. Hence, the inter-wall distance $l_{\text {wall }}$, which can be different in each of the three dimensions, enters the list of physical variables that affect polymer properties. Unavoidably, there is a relative arbitrariness in the comparison between a system possessing periodic boundary conditions and a confined one. Based on this for the case of 1-d confinement of cubic cells, and in order to define the basic set of parameters, we use the density of the corresponding periodic (bulk) system along with the inter-wall distance.

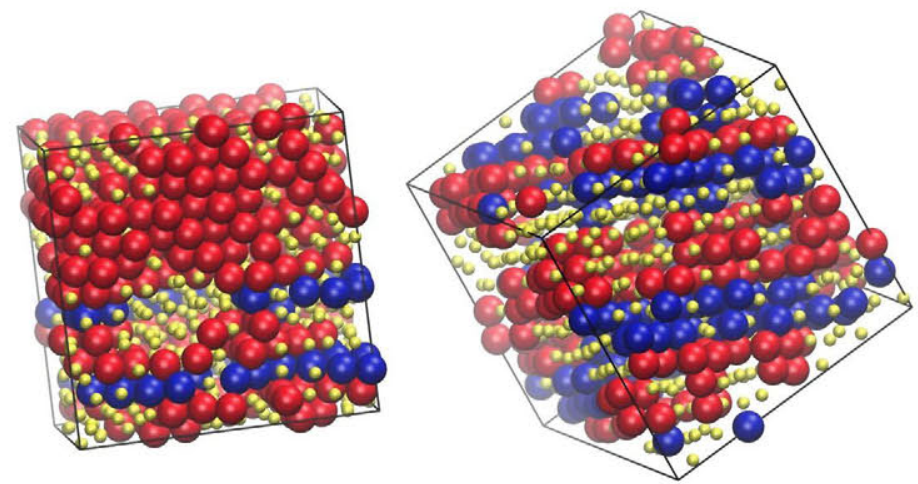

We should state here that while the effect of confinement has been studied extensively in the literature, the off-lattice simulation of confined and dense systems is non-trivial even for monomeric hard spheres especially with respect to the phase behaviour (Fortini and Dijkstra, 2006; Veshnev and Nurlygayanov, 2011). For polymer packings, the situation is even more complicated as the inter-wall distance (which in general can be different in each direction) is compared not only with the characteristic length of the system (the sphere diameter) but also with the average chain size (as quantified for example by the radius of gyration).

The computational efficiency of the MC protocol in equilibrating the long-range chain characteristics is quantified by the decay of the orientational autocorrelation function of the end-toend unit vector $\langle\mathbf{u}(t) \cdot \mathbf{u}(0)\rangle$, as a function of time, $t$, measured here in MC steps. $\mathbf{u}(t)$ is the chain end-to-end unit vector, at time $t$, and brackets denote averaging over all chains and recorded frames. The faster $\langle\mathbf{u}(t) \cdot \mathbf{u}(0)\rangle$ drops to zero, the faster the chain system loses the memory of the initial configuration. Fig. 14 shows the time decay of $\langle\mathbf{u}(t) \cdot \mathbf{u}(0)\rangle$ for the $N=100$ system in the bulk $(\varphi=0.40$ and 0.60 ) and under $1-d$ confinement. It is clear that chain-connectivity-altering moves (here the SEB and SIEB) provide very rapid and robust long-range equilibration as in the case of chemically more complex polymer systems. In parallel, local relaxation is undertaken by the rest of the MC moves, executed in the adaptive bias pattern, which show high

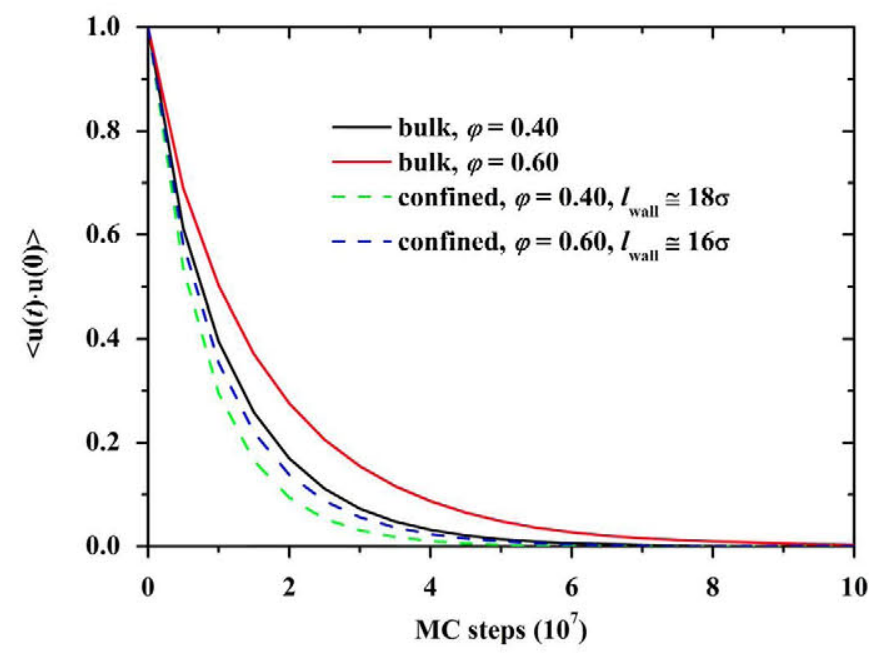

Fig. 14. Orientational autocorrelation function of the end-to-end unit vector $\langle\mathbf{u}(t) \cdot \mathbf{u}(0)\rangle$ as a function of MC steps for the $N=100$ system in the bulk $(\varphi=0.40$ and 0.60 ) and under flat impenetrable walls in one dimension. $l_{\text {wall }}$ denotes the distance between the wall boundaries in the case of confinement.

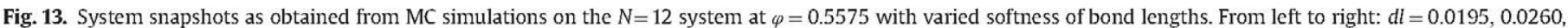

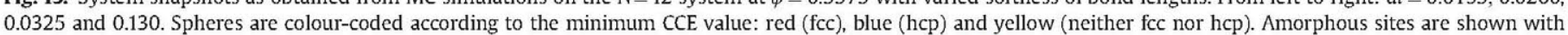

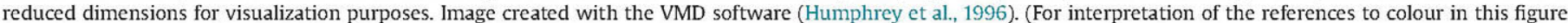
legend, the reader is referred to the web version of this article.)
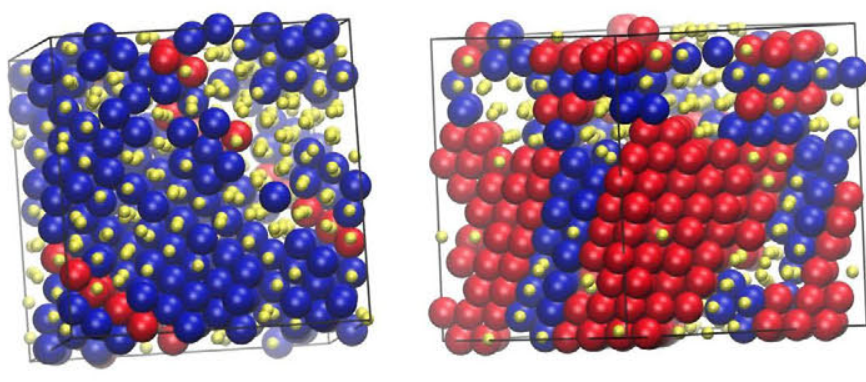
efficiency. This is demonstrated by the ability of the MC code to track crystallization even at very high volume fractions (as established in the previous section). The rate of local versus global (chain) equilibration can be tuned by altering the corresponding attempt probabilities of the local and chainconnectivity-altering MC moves.

Packing density has only a small effect on long-range equilibration, even at the volume fractions of $\varphi=0.40$ and 0.60 which lie in the marginal and concentrated regimes, respectively. Even in the latter case, close to the MRJ state, the present MC scheme requires only a few millions of steps (corresponding to minutes in wall-clock time) to provide long-range equilibration. Additionally, the trends with respect to the efficiency of the MC algorithm appear to be unaffected by the presence of the impenetrable wall in one dimension. In fact the confined system at the same density as the bulk analogue shows more rapid relaxation than the periodic one. Thus, the proposed MC scheme can be reliably applied to model athermal polymers under spatial constraints with the ultimate goal to identify the effect of extreme confinement on polymer jamming.

The presence of wall boundaries in one dimension renders the system highly non-isotropic. This in turn, affects all structural properties which either have to be defined in reference of individual dimensions, or have to be reported as a function of the distance to the confining walls. Prominent among the affected quantities is the local monomer density (concentration) which becomes highly anisotropic and heterogeneous under confinement. This is vividly shown in Fig. 15 for the $N=100$ system at three different packing densities (corresponding to the bulk periodic analogue) with varied inter-wall distances. In order to understand the level of confinement we also report the average dimensions of the chains, as quantified by the root mean square radius of gyration. Anisotropy in concentration is immediately apparent by comparing the density profiles at each dimension. Furthermore, the presence of the wall creates heterogeneity in the density profile, which is distinctly different near the walls than far from them. Characteristic concentration minima and maxima near the walls can be attributed to specific chain conformations in combination with the impenetrability of the wall surface. As density increases, the presence of the wall, even if there is no short- or long-range interaction with the chain monomers, affects the spatial distribution of the latter at ranges that exceed the average size of the chains. At high volume fractions density profile adopts a regular pattern indicative of ordering, in contrast to the uniform one of the corresponding bulk system which remains in the amorphous state at the same packing density. We can thus conclude that by tuning the level of confinement one can control the phase behaviour of dense polymer packings.

\section{Conclusions}

We have presented the main results obtained from extensive Monte Carlo (MC) simulations on freely-jointed chains of hard spheres of uniform size. Through an effective algorithm, based on chain-connectivity altering moves, we are able to identify the maximally random jammed (MRJ) state of athermal polymer packings and compare its salient characteristics with the ones exhibited by the corresponding state of monomeric analogues. By exploring the full concentration range, we can accurately identify the characteristic scaling regimes of chain size with packing density, the corresponding scaling exponents and the transition thresholds. Through geometric algorithms the parent chain configurations are mapped into the underlying primitive path (PP) network of inter-chain topological constraints in the form of entanglements. The corresponding scaling laws can be extracted also for entanglements while a connection is revealed between entanglements and knots, valid from dilute systems up to nearly jammed ones.

As the length of the simulations is increased we observe the spontaneous, entropically-driven crystallization of athermal, initially random packings. The phase (disorder-order) transition shares a wealth of similarities with the one exhibited by monoatomic counterparts; bond constraints related to tangency condition seem to frustrate polymer crystallization near the melting transition.

Finally, we presented preliminary results from MC simulations on spatially-constrained polymer systems which are confined, through flat impenetrable walls, in at least one dimension. Comparison can thus be made on how the level of confinement affects the static properties and structure of macromolecules as well as their ability to crystallize.

By being complex and anisotropic systems, athermal polymers constitute a class on their own in the general topic of atomic/ molecular and particulate packing. Due to their chemical simplicity hard-sphere chains provide an excellent statistical mechanical model to test, verify and guide theoretical concepts related to polymer physics. In a coarse-to-fine modelling approach, athermal polymers can act as the ground case in simulations of progressively finer chemical detail.

In the present work we have identified the packing properties of fully flexible linear athermal chains of monomers with uniform size in the bulk and under confinement. Current and future modelling extensions focus on (i) studying the effect of chain architecture on jamming, (ii) the simulation of polymer blends with binary distribution of sphere size, (iii) modelling conditions of extreme confinement with the inter-wall distance being as close as possible to sphere diameter (Termonia, 2011), (iv) the simulation of terminally-anchored polymers in complex geometries
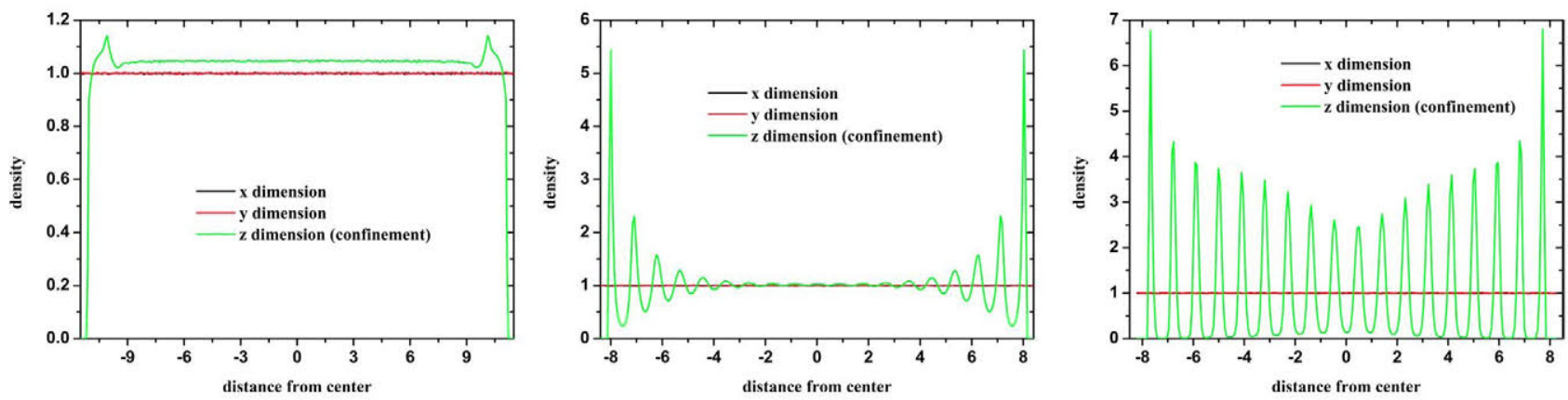

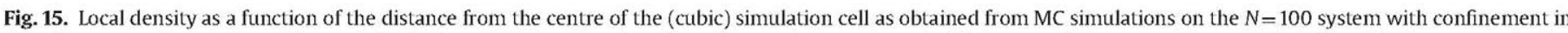

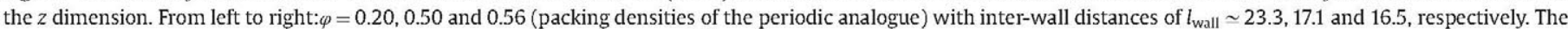
corresponding values for the root mean square radius of gyration are $\left\langle R_{g}^{2}\right\rangle^{1 / 2} \simeq 6.29,5.04$ and 4.75 . 
(Benetatos, 2014; Daoulas et al., 2005, 2002; Hammer and Kantor 2014; Maghrebi et al., 2012), (v) analysing the effect of chain stiffness on jamming and phase behaviour, and (vi) the simulation of polymer-colloid mixtures (Dijkstra et al., 2006).

From the application perspective, dense packings of polymeric systems are particular relevant in a wide range of physical and chemical systems: from colloids and liquid crystals to complex synthetic interfaces and biomolecules.

\section{Acknowledgements}

We deeply appreciate fruitful discussions and collaborations with Professors Martin Kröger (ETH, Switzerland), Robert Hoy (University of South Florida, USA), Marc Mansfield (Steven Institute of Technology, USA), Juan J. de Pablo (University of Chicago, USA), Cameron Abrams (Drexel University, USA), J. Shen (Tongji University, PRC), Dr. Chen Wu (Harbin Institute of Technology, PRC) and Pablo Ramos (Polytechnic University of Madrid, Spain). N.C.K. acknowledges support through projects "Ramon y Cajal" (RYC-2009-05413), "I3" and "MAT2010-15482" of the Spanish Ministry of Economy and Competitiveness (MINECO). K.F. acknowledges support through projects "Ramon y Cajal" (RYC2010-06804) and "MAT2011-24834" of MINECO. Authors thankfully acknowledge the computer resources, technical expertise and assistance provided by the Centro de Computacion y Visualizacion de Madrid (CeSViMa).

\section{References}

Alder, B.J., Wainwright, T.E., 1957. Phase transition for a hard sphere system. J. Chem. Phys. 27, 1208-1209.

Allen, M.P., Tildesley, D.J., 1987. Computer Simulation of Liquids. Oxford University Press, New York.

Anikeenko, A.V., Medvedev, N.N., 2007. Polytetrahedral nature of the dense disordered packings of hard spheres. Phys. Rev. Lett. 98, 235504.

Anikeenko, A.V., Medvedev, N.N., Aste, T., 2008. Structural and entropic insight into the nature of the random-close-packing limit. Phys. Rev. E 77, 031101.

Anikeenko, A.V., Medvedev, N.N., Bezrukov, A., Stoyan, D., 2007. Observation of fivefold symmetry structures in computer models of dense packing of hard spheres. J. Non-Cryst. Solids 353, 3545-3549.

Anogiannakis, S.D., Tzoumanekas, C., Theodorou, D.N., 2012. Microscopic description of entanglements in polyethylene networks and melts strong, weak, pairwise, and collective attributes. Macromolecules 45, 9475-9492.

Aste, T., 2005. Variations around disordered close packing. J. Phys.-Condes. Matter 17, S2361-S2390.

Aste, T., Saadatfar, M., Senden, T.J., 2005. Geometrical structure of disordered sphere packings. Phys. Rev, E 71, 061302.

Auer, S., Frenkel, D., 2001. Prediction of absolute crystal-nucleation rate in hardsphere colloids. Nature 409, 1020-1023.

Bagley, B.G., 1970. 5-fold pseudosymmetry. Nature 225, 1040 (\&).

Ballesta, P., Duri, A., Cipelletti, L., 2008. Unexpected drop of dynamical heterogeneities in colloidal suspensions approaching the jamming transition. Nat Phys. 4, 550-554.

Benetatos, P., 2014. Crosslink-induced shrinkage of grafted gaussian chains. Phys Rev. E 89, 042602

Bernal, J.D., 1959. Geometrical approach to the structure of liquids. Nature 183 141-147.

Bernal, J.D. 1960. Geometry of the structure of monatomic liquids. Nature 185 $68-70$

Bernal, J.D., Finney, J.L., 1967. Random close-packed hard-sphere model.2. Geomery of random packing of hard spheres. Discuss. Faraday Soc, 43, 62-69.

Bernal, J.D., Mason, J., 1960. Co-ordination of randomly packed spheres. Nature 188 910-911.

Bolhuis, P.G., Frenkel, D., Mau, S.C., Huse, D.A., 1997. Entropy difference between crystal phases. Nature 388, 235-236.

Borchard-Ott, W., 2002. Kristallographie. Springer Verlag, Berlin.

Brown, E., Nasto, A., Athanassiadis, A.G., Jaeger, H.M., 2012. Strain stiffening in random packings of entangled granular chains. Phys. Rev. Lett. 108, 108302.

Corwin, E.I., Jaeger, H.M., Nagel, S.R., 2005. Structural signature of jamming in granular media. Nature 435, 1075-1078.

Cotton, J.P., Nierlich, M., Boue, F, Daoud, M., Farnoux, B. Jannink, G, Duplessix, R, Picot, C., 1976. Experimental-determination of temperature-concentration diagram of flexible polymer-solutions by neutron-scattering. J. Chem. Phys. $65,1101-1108$

Chaikin, P.M. Lubensky, T.C., 2000. Principles of Condensed Matter Physics. Cambridge University Press, Cambridge.
Cheng, Z.D., Chaikin, P.M., Zhu, J.X., Russel, W.B., Meyer, W.V., 2002. Crystallization kinetics of hard spheres in microgravity in the coexistence regime: interactions between growing crystallites. Phys. Rev. Lett. 88, 015501.

Daoud, M., Cotton, J.P., Farnoux, B., Jannink, G., Sarma, G., Benoit, H., Duplessix, R., Picot, C., Gennes, P.G.D., 1975. Solutions of flexible polymers - neutron experiments and interpretation. Macromolecules 8, 804-818.

Daoulas, K.C., Harmandaris, V.A., Mavrantzas, V.G., 2005. Detailed atomistic simulation of a polymer melt/solid interface: structure, density, and conformation of a thin film of polyethylene melt adsorbed on graphite. Macromolecules $38,5780-5795$.

Daoulas, K.C., Terzis, A.F., Mavrantzas, V.G., 2002. Detailed atomistic Monte Carlo simulation of grafted polymer melts. 1 . Thermodynamic and conformational properties. J. Chem. Phys, 116, 11028-11038.

de Pablo, J.J., Laso, M., Suter, U.W., 1992a. Estimation of the chemical-potential of chain molecules by simulation. J. Chem. Phys. 96, 6157-6162.

de Pablo, J.J., Laso, M., Suter, U.W., 1992b. Simulation of polyethylene above and below the melting-point. J. Chem. Phys. 96, 2395-2403.

deGennes, P.G., 1980. Scaling Concepts in Polymer Physics. Cornell University Press, Ithaca.

Dijkstra, M., van Roij, R., Roth, R., Fortini, A., 2006. Effect of many-body interactions on the bulk and interfacial phase behavior of a model colloid-polymer mixture. Phys. Rev. E 73, 041404.

Dodd, L.R., Boone, T.D., Theodorou, D.N., 1993. A concerted rotation algorithm for atomistic Monte-Carlo simulation of polymer melts and glasses. Mol. Phys. 78, 961-996.

Doi, M., Edwards, S.F., 1988. The Theory of Polymer Dynamics. Clarendon Press, Oxford.

Donev, A., Cisse, 1., Sachs, D., Variano, E., Stillinger, F.H., Connelly, R., Torquato, S., Chaikin, P.M., 2004a. Improving the density of jammed disordered packings using ellipsoids. Science 303, 990-993.

Donev, A., Stillinger, F.H., Torquato, S., 2005a. Unexpected density fluctuations in jammed disordered sphere packings. Phys. Rev. Lett. 95, 090604.

Donev, A., Stillinger, F.H., Torquato, S., 2006. Do binary hard disks exhibit an ideal glass transition? Phys. Rev. Lett. 96, 225502

Donev, A., Torquato, S., Stillinger, F.H., 2005b. Pair correlation function characteristics of nearly jammed disordered and ordered hard-sphere packings. Phys. Rev. E 71, 011105.

Donev, A., Torquato, S., Stillinger, F.H., Connelly, R., 2004b. Jamming in hard sphere and disk packings. J. Appl. Phys. 95, 989-999.

Edwards, S.F., 1966. Theory of polymer solutions at intermediate concentration. Proc. Phys. Soc. 88, 265-280.

Eldridge, M.D., Madden, P.A., Frenkel, D., 1993. Entropy-driven formation of a superlattice in a hard-sphere binary mixture. Nature $365,35-37$

Escobedo, F.A., de Pablo, J.J., 1995. Extended continuum configurational bias MonteCarlo methods for simulation of flexible molecules. J. Chem. Phys. 102, 2636-2652.

Everaers, R., 2012. Topological versus rheological entanglement length in primitivepath analysis protocols, tube models, and slip-link models. Phys. Rev. E 86, 022801.

Everaers, R., Sukumaran, S.K., Grest, G.S., Svaneborg, C., Sivasubramanian, A., Kremer, K., 2004. Rheology and microscopic topology of entangled polymeric liquids. Science 303, 823-826.

Farnoux, B., Boue, F., Cotton, J.P., Daoud, M., Jannink, G., Nierlich, M., Degennes, P.G., 1978. Crossover in polymer-solutions. J. Phys.-Paris 39, 77-86.

Fetters, L.J., Lohse, D.J., Milner, S.T., Graessley, W.W., 1999. Packing length influence in linear polymer melts on the entanglement, critical, and reptation molecular weights. Macromolecules 32, 6847-6851.

Finney, J.L., 1970. Random packings and structure of simple liquids.1. Geometry of random close packing. Proc. R. Soc. London Ser. A 319, 479-493.

Fleer, G.J., Cohen Stuart, M.A., Scheutjens, J.M.H.M., Cosgrove, T., Vincent, B., 1993. Polymers at Intefaces. Chapman and Hall, London.

Flory, P.J., 1989. Statistical Mechanics of Chain Molecules. Hanser-Verlag, Munchen.

Fortini, A., Dijkstra, M., 2006. Phase behaviour of hard spheres confined between parallel hard plates: manipulation of colloidal crystal structures by confinement. J. Phys.-Condes. Matter 18, L371-L378.

Foteinopoulou, K., Karayiannis, N.C., Laso, M., Kroger, M., 2009. Structure, dimensions, and entanglement statistics of long linear polyethylene chains. J. Phys. Chem. B 113, 442-455.

Foteinopoulou, K., Karayiannis, N.C., Laso, M., Kroger, M., Mansfield, M.L., 2008. Universal scaling, entanglements, and knots of model chain molecules. Phys. Rev. Lett. 101, 265702

Foteinopoulou, K., Karayiannis, N.C., Mavrantzas, V.G., Kroger, M., 2006. Primitive path identification and entanglement statistics in polymer melts: results from direct topological analysis on atomistic polyethylene models. Macromolecules $39,4207-4216$.

Frenkel, D., 1988. Structure of hard-core models for liquid-crystals. J. Phys. Chem. 92, 3280-3284.

Frenkel, D., 1994. Computation challenges in complex liquids - entropy-driven phase-transitions. Future Gener. Comp. Syst. 10, 207-212.

Frenkel, D., 1999. Entropy-driven phase transitions. Physica A 263, 26-38.

Frenkel, D., 2000. Perspective on "the effect of shape on the interaction of colloidal particles" - onsager 1 (1949) ann ny acad sci 51: 627. Theor. Chem. Acc. 103, 212-213.

Frenkel, D., Lekkerkerker, H.N.W., Stroobants, A., 1988. Thermodynamic stability of a smectic phase in a system of hard-rods. Nature 332, 822-823.

Frenkel, D., Smit, B., 2002. Understanding Molecular Simulation: From Algorithms to Applications, second ed. Academic Pr 
Giacovazzo, C., Monaco, H.L., Artioli, G., Viterbo, D., Ferraris, G., Gilli, G., Zanotti, G., Gatti, M., 2005. Fundamentals of Crystallography. Oxford Science, Oxford.

Goodrich, C.P., Liu, A.J., Nagel, S.R., 2012. Finite-size scaling at the jamming transition. Phys. Rev. Lett. 109, 095704.

Haji-Akbari, A., Chen, E.R., Engel, M., Glotzer, S.C., 2013. Packing and self-assembly of truncated triangular bipyramids. Phys. Rev. E 88, 012127.

Haji-Akbari, A., Engel, M., Glotzer, S.C., 2011a. Degenerate quasicrystal of hard triangular bipyramids. Phys. Rev. Lett. 107, 215702.

Haji-Akbari, A., Engel, M., Glotzer, S.C., 2011b. Phase diagram of hard tetrahedra. J. Chem. Phys. 135, 194101.

Haji-Akbari, A., Engel, M., Keys, A.S., Zheng, X., Petschek, R.G., Palffy-Muhoray, P., Glotzer, S.C., 2009. Disordered, quasicrystalline and crystalline phases of densely packed tetrahedra. Nature 462, 773-U791.

Hales, T.C., 2005. A proof of the kepler conjecture. Ann. Math. 162, 1065-1185.

Hales, T.C., Harrison, J., McLaughlin, S., Nipkow, T., Obua, S., Zumkeller, R., 2010. A revision of the proof of the Kepler conjecture. Discret. Comput. Geom. 44, 1-34.

Hammer, Y., Kantor, Y., 2014. Ideal polymers near scale-free surfaces. Phys. Rev. E $89,022601$.

Harland, J.L., vanMegen, W., 1997. Crystallization kinetics of suspensions of hard colloidal spheres. Phys. Rev. E 55, 3054-3067.

Haslam, A.J., Jackson, G., McLeish, T.C.B., 1999. An investigation of the shape and crossover scaling of flexible tangent hard-sphere polymer chains by Monte Carlo simulation. J. Chem. Phys. 111, 416-428.

He, Y.M., Olivier, B., Ackerson, B.J., 1997. Morphology of crystals made of hard spheres. Langmuir 13, 1408-1412.

Henderson, S.I., van Megen, W., 1998. Metastability and crystallization in suspensions of mixtures of hard spheres. Phys. Rev. Lett. 80, 877-880.

Honeycutt, J.D., Andersen, H.C., 1987. Molecular-dynamics study of melting and freezing of small Lennard-Jones clusters. J. Phys. Chem. 91, 4950-4963.

Hoy, R.S., Foteinopoulou, K., Kroger, M., 2009. Topological analysis of polymeric melts: chain-length effects and fast-converging estimators for entanglement length. Phys. Rev. E 80, 031803.

Hoy, R.S., Karayiannis, N.C., 2013. Simple model for chain packing and crystallization of soft colloidal polymers. Phys. Rev. E 88, 012601.

Humphrey, W., Dalke, A., Schulten, K., 1996. Vmd: visual molecular dynamics. J. Mol. Graph. 14, 33-38.

Jiao, Y., Stillinger, F.H., Torquato, S., 2009. Optimal packings of superballs. Phys. Rev. E 79, 041309.

Jiao, Y., Torquato, S., 2011. Maximally random jammed packings of platonic solids: hyperuniform long-range correlations and isostaticity. Phys. Rev. E 84, 041309

Jullien, R., Jund, P., Caprion, D., Quitmann, D., 1996. Computer investigation of longrange correlations and local order in random packings of spheres. Phys. Rev. E $54,6035-6041$.

Kamio, K., Moorthi, K., Theodorou, D.N., 2007. Coarse grained end bridging monte carlo simulations of poly(ethylene terephthalate) melt. Macromolecules 40, 710-722.

Karayiannis, N.C., Foteinopoulou, K., Abrams, C.F., Laso, M., 2010. Modeling of crystal nucleation and growth in athermal polymers: self-assembly of layered nanomorphologies. Soft Matter 6, 2160-2173.

Karayiannis, N.C., Foteinopoulou, K., Laso, M., 2009a. Contact network in nearly jammed disordered packings of hard-sphere chains. Phys. Rev. E 80, 011307.

Karayiannis, N.C., Foteinopoulou, K., Laso, M., 2009b. The characteristic crystallographic element norm: a descriptor of local structure in atomistic and particulate systems. J. Chem. Phys. 130, 074704.

Karayiannis, N.C., Foteinopoulou, K., Laso, M., 2009c. Entropy-driven crystallization in dense systems of athermal chain molecules. Phys. Rev. Lett. 103, 045703.

Karayiannis, N.C., Foteinopoulou, K., Laso, M., 2009d. The structure of random packings of freely jointed chains of tangent hard spheres. J. Chem. Phys. 130, 164908.

Karayiannis, N.C., Foteinopoulou, K., Laso, M., 2013a. Jamming and crystallization in athermal polymer packings. Philos. Mag. 93, 4108-4131.

Karayiannis, N.C., Foteinopoulou, K., Laso, M., 2013b. Spontaneous crystallization in athermal polymer packings. Int. J. Mol. Sci. 14, 332-358.

Karayiannis, N.C., Giannousaki, A.E., Mavrantzas, V.G., Theodorou, D.N., 2002a. Atomistic Monte Carlo simulation of strictly monodisperse long polyethylene melts through a generalized chain bridging algorithm. J. Chem. Phys. 117, 5465-5479.

Karayiannis, N.C., Kroger, M., 2009. Combined molecular algorithms for the generation, equilibration and topological analysis of entangled polymers: methodology and performance. Int. J. Mol. Sci. 10, 5054-5089.

Karayiannis, N.C., Laso, M., 2008a. Dense and nearly jammed random packings of freely jointed chains of tangent hard spheres. Phys. Rev. Lett. 100, 050602.

Karayiannis, N.C., Laso, M., 2008b. Monte Carlo scheme for generation and relaxation of dense and nearly jammed random structures of freely jointed hard-sphere chains. Macromolecules 41, 1537-1551.

Karayiannis, N.C., Malshe, R., de Pablo, J.J., Laso, M., 2011. Fivefold symmetry as an inhibitor to hard-sphere crystallization. Phys. Rev. E 83, 061505.

Karayiannis, N.C., Malshe, R., Kroger, M., de Pablo, J.J., Laso, M., 2012. Evolution of fivefold local symmetry during crystal nucleation and growth in dense hardsphere packings. Soft Matter 8, 844-858.

Karayiannis, N.C., Mavrantzas, V.G., Theodorou, D.N., 2002b. A novel Monte Carlo scheme for the rapid equilibration of atomistic model polymer systems of precisely defined molecular architecture. Phys. Rev. Lett. 88, 105503.

Kawasaki, T., Tanaka, H., 2010. Formation of a crystal nucleus from liquid. Proc. Natl. Acad. Sci. USA 107, 14036-14041.

Keys, A.S., Abate, A.R., Glotzer, S.C., Durian, D.J., 2007. Measurement of growing dynamical length scales and prediction of the jamming transition in a granular material. Nat Phys, $3,260-264$.
Khaliullin, R.N., Schieber, J.D., 2009. Self-consistent modeling of constraint release in a single-chain mean-field slip-link model. Macromolecules 42, 7504-7517.

Koniaris, K., Muthukumar, M., 1991. Knottedness in ring polymers. Phys. Rev. Lett. 66, 2211-2214

Kroger, M., 1999. Efficient hybrid algorithm for the dynamic creation of wormlike chains in solutions, brushes, melts and glasses. Comput. Phys. Commun. 118, $278-298$.

Kroger, M., 2004. Simple models for complex nonequilibrium fluids. Phys. Rep.-Rev. Sect. Phys. Lett. 390, 453-551.

Kroger, M., 2005. Shortest multiple disconnected path for the analysis of entanglements in two- and three-dimensional polymeric systems. Comput. Phys. Commun. 168, 209-232.

Kroger, M., Muller, M., Nievergelt, J., 2003. A geometric embedding algorithm for efficiently generating semiflexible chains in the molten state. Comput. Model. Eng. Sci. 4, 559-569.

Laso, M., de Pablo, J.J., Suter, U.W., 1992. Simulation of phase-equilibria for chain molecules. J. Chem. Phys. 97, 2817-2819.

Laso, M., Karayiannis, N.C., 2008. Flexible chain molecules in the marginal and concentrated regimes: universal static scaling laws and cross-over predictions. J. Chem. Phys. 128, 174901.

Laso, M., Karayiannis, N.C., Foteinopoulou, K., Mansfield, M.L., Kroger, M., 2009 Random packing of model polymers: local structure, topological hindrance and universal scaling. Soft Matter 5, 1762-1770.

Laso, M., Karayiannis, N.C., Muller, M., 2006. Min-map bias monte carlo for chain molecules: biased monte carlo sampling based on bijective minimum-tominimum mapping. J. Chem. Phys. 125, 164108.

Leocmach, A., Tanaka, H., 2012. Roles of icosahedral and crystal-like order in the hard spheres glass transition. Nat. Commun. 3, 974

Likhtman, A.E., 2005. Single-chain slip-link model of entangled polymers: simultaneous description of neutron spin-echo, rheology, and diffusion. Macromolecules $38,6128-6139$

Likhtman, A.E., McLeish, T.C.B., 2002. Quantitative theory for linear dynamics of linear entangled polymers. Macromolecules 35, 6332-6343.

Lu, K., Brodsky, E.E., Kavehpour, H.P., 2008. A thermodynamic unification of jamming. Nat. Phys. 4, 404-407.

Maghrebi, M.F., Kantor, Y., Kardar, M., 2012. Polymer-mediated entropic forces between scale-free objects. Phys. Rev. E 86, 061801.

Malanoski, A.P., Monson, P.A., 1997. The high density equation of state and solidfluid equilibrium in systems of freely jointed chains of tangent hard spheres. J. Chem. Phys. 107, 6899-6907.

Mansfield, M.L., 2007a. Development of knotting during the collapse transition of polymers. J. Chem. Phys. 127, 244902

Mansfield, M.L., 2007b. Efficient knot group identification as a tool for studying entanglements of polymers. J. Chem. Phys. 127, 244901.

Marrucci, G., 1996. Dynamics of entanglements: a nonlinear model consistent with the Cox-Merz rule. J. Non-Newton. Fluid Mech. 62, 279-289.

Marrucci, G., lanniruberto, G., 2004. Interchain pressure effect in extensional flows of entangled polymer melts. Macromolecules 37, 3934-3942.

Masubuchi, Y., lanniruberto, G., Greco, F., Marrucci, G., 2003. Entanglemen molecular weight and frequency response of sliplink networks. J. Chem. Phys $119,6925-6930$.

Mavrantzas, V.G., Boone, T.D., Zervopoulou, E., Theodorou, D.N., 1999. End-bridging monte carlo: a fast algorithm for atomistic simulation of condensed phases of long polymer chains. Macromolecules 32, 5072-5096.

McBride, C., Vega, C., MacDowell, L.G., 2001. Isotropic-nematic phase transition: Influence of intramolecular flexibility using a fused hard sphere model. Phys. Rev. E 64, 011703.

McLeish, T.C.B., Larson, R.G., 1998. Molecular constitutive equations for a class of branched polymers: the pom-pom polymer. J. Rheol. 42, 81-110.

Ni, R., Dijkstra, M., 2013. Effect of bond length fluctuations on crystal nucleation of hard bead chains. Soft Matter 9, 365-369.

O'Hern, C.S., Langer, S.A., Liu, A.J., Nagel, S.R., 2002. Random packings of frictionless particles. Phys. Rev. Lett. 88, 075507.

O'Hern, C.S., Silbert, L.E., Liu, A.J., Nagel, S.R., 2003. Jamming at zero temperature and zero applied stress: the epitome of disorder. Phys. Rev. E 68, 011306.

O'Malley, B., Snook, I., 2003. Crystal nucleation in the hard sphere system. Phys. Rev. Lett. 90, 085702

Onsager, L., 1949. The effects of shape on the interaction of colloidal particles. Ann. NY Acad. Sci. 51, 627-659.

Ostwald, W., 1897. Studien über die Bildung and Umwandlung fester Körper. Z. Phys. Chem. 22, 289-330.

Palberg, T., Stipp, A., Bartsch, E., 2009. Unusual crystallization kinetics in a hard sphere colloid-polymer mixture. Phys. Rev. Lett. 102, 038302.

Panagiotou, E., Kroger, M., Millett, K.C., 2013. Writhe and mutual entanglemen combine to give the entanglement length. Phys. Rev. E 88, 062604.

Pant, P.V.K., Theodorou, D.N., 1995. Variable connectivity method for the atomistic Monte-Carlo simulation of polydisperse polymer melts. Macromolecules 28 7224-7234.

Pusey, P.N., Vanmegen, W., 1986. Phase-behavior of concentrated suspensions of nearly hard colloidal spheres. Nature 320, 340-342.

Pusey, P.N., Vanmegen, W., Bartlett, P., Ackerson, B.J., Rarity, J.G., Underwood, S.M., 1989. Structure of crystals of hard colloidal spheres. Phys. Rev. Lett. 63, 2753-2756.

Pusey, P.N., Zaccarelli, E., Valeriani, C., Sanz, E., Poon, W.C.K., Cates, M.E., 2009. Hard spheres: crystallization and glass formation. Philos. Trans. R. Soc. A-Math. Phys. Eng. Sci. 367, 4993-5011. 
Qin, J., Milner, S.T., 2011. Counting polymer knots to find the entanglement length. Soft Matter 7, 10676-10693.

Ramirez, J., Sukumaran, S.K., Likhtman, A.E., 2007. Hierarchical modeling of entangled polymers. Macromol. Symp. 252, 119-129.

Rawdon, E., Dobay, A., Kern, J.C., Millett, K.C., Piatek, M., Plunkett, P., Stasiak, A. 2008a. Scaling behavior and equilibrium lengths of knotted polymers. Macromolecules 41, 4444-4451.

Rawdon, E.J., Kern, J.C., Piatek, M., Plunkett, P., Stasiak, A., Millett, K.C., 2008b. Effect of knotting on the shape of polymers. Macromolecules 41, 8281-8287.

Rintoul, M.D., Torquato, S., 1996. Metastability and crystallization in hard-sphere systems. Phys. Rev. Lett. 77, 4198-4201.

Russo, J., Tanaka, H., 2012. The microscopic pathway to crystallization in supercooled liquids. Sci. Rep. 2, 505.

Scott, G.D., Kilgour, D.M., 1969. Density of random close packing of spheres. J. Phys. D-Appl. Phys. 2, 863 (\&).

Scott, G.D., Knight, K.R., Bernal, J.D., Mason, J., 1962. Radial distribution of random close packing of equal spheres. Nature 194, 956-957.

Schilling, T., Schope, H.J., Oettel, M., Opletal, G., Snook, I., 2010. Precursor-mediated crystallization process in suspensions of hard spheres. Phys. Rev. Lett. 105, 025701.

Shanbhag, S., Larson, R.G., 2005. Chain retraction potential in a fixed entanglement network. Phys. Rev. Lett. 94, 076001.

Siepmann, J.1., Frenkel, D., 1992. Configurational bias monte-carlo - a new sampling scheme for flexible chains. Mol. Phys. 75, 59-70.

Steenbakkers, R.J.A., Tzoumanekas, C., Li, Y., Liu, W.K., Kroger, M., Schieber, J.D. 2014. Primitive-path statistics of entangled polymers: mapping multi-chain simulations onto single-chain mean-field models. New J. Phys. 16, 015027.

Steinhardt, P.J., Nelson, D.R., Ronchetti, M., 1983. Bond-orientational order in liquids and glasses. Phys. Rev. B 28, 784-805.

Stephanou, P.S., Baig, C., Mavrantzas, V.G., 2011. Projection of atomistic simulation data for the dynamics of entangled polymers onto the tube theory: calculation of the segment survival probability function and comparison with modern tube models. Soft Matter 7, 380-395.

Stroobants, A., Lekkerkerker, H.N.W., Frenkel, D., 1987. Evidence for one-dimensional, two-dimensional, and 3-dimensional order in a system of hard parallel spherocylinders. Phys. Rev. A 36, 2929-2945.

Termonia, Y., 2011. Two-dimensional nanometric confinement of entangled polymer melts. Polymer 52, 5193-5196.

Theodorou, D.N., Tzoumanekas, C., 2006. From atomistic simulations to slip-link models of entangled polymer melts: hierarchical strategies for the prediction of rheological properties. Curr. Opin. Solid State Mater. Sci. 10, 61-72.

Tian, Z.A., Dong, K.J., Yu, A.B., 2014. Structural evolution in the packing of uniform spheres. Phys. Rev. E 89, 032202.
Torquato, S., Jiao, Y., 2009. Dense packings of the platonic and archimedean solids. Nature 460, 876 (U109).

Torquato, S., Truskett, T.M., Debenedetti, P.G., 2000. Is random close packing of spheres well defined? Phys. Rev. Lett. 84, 2064-2067.

Tzoumanekas, C., Lahmar, F., Rousseau, B., Theodorou, D.N., 2009. Onset of entanglements revisited. Topological analysis. Macromolecules 42, 7474-7484.

Tzoumanekas, C., Theodorou, D.N., 2006. Topological analysis of linear polymer melts: a statistical approach. Macromolecules 39, 4592-4604.

Uhlherr, A., Doxastakis, M., Mavrantzas, V.G., Theodorou, D.N., Leak, S.J., Adam, N.E., Nyberg, P.E., 2002. Atomic structure of a high polymer melt. Europhys. Lett. 57, 506-511.

Veerman, J.A.C., Frenkel, D., 1991. Relative stability of columnar and crystalline phases in a system of parallel hard spherocylinders. Phys. Rev. A 43, 4334-4343.

Vega, C., McBride, C., MacDowell, L.G., 2001. Liquid crystal phase formation for the linear tangent hard sphere model from monte carlo simulations. J. Chem. Phys. $115,4203-4211$.

Veshnev, V.P., Nurlygayanov, T.A., 2011. Crystallization of hard spheres near wall. Tech. Phys. Lett. 37, 863-865.

Vutukuri, H.R., Demirors, A.F., Peng, B., van Oostrum, P.D.J., Imhof, A., van Blaaderen, A., 2012. Colloidal analogues of charged and uncharged polymer chains with tunable stiffness. Angew. Chem.-Int. Ed. 51, 11249-11253.

Wang, Z.W., Likhtman, A.E., Larson, R.G., 2012. Segmental dynamics in entangled linear polymer melts. Macromolecules 45, 3557-3570.

Woodcock, L.V., 1997. Entropy difference between the face-centred cubic and hexagonal close-packed crystal structures. Nature 385, 141-143.

Wu, C., Karayiannis, N.C., Laso, M., Qu, D., Luo, Q., Shen, J., 2014. A metric to gauge local distortion in metallic glasses and supercooled liquids. Acta Mater. 72 229-238.

Xu, N., Blawzdziewicz, J., O'Hern, C.S., 2005. Random close packing revisited: ways to pack frictionless disks. Phys. Rev. E 71, 061306.

Yethiraj, A., Dickman, R., 1992. Local-structure of model polymeric fluids - hardsphere chains and the 3-dimensional fluctuating bond model. J. Chem. Phys. 97, 4468-4475.

Yethiraj, A., Hall, C.K., 1992. Monte-carlo simulations and integral-equation theory for microscopic correlations in polymeric fluids. J. Chem. Phys. 96, 797-807.

Zaccarelli, E., Valeriani, C., Sanz, E., Poon, W.C.K., Cates, M.E., Pusey, P.N., 2009. Crystallization of hard-sphere glasses. Phys. Rev. Lett. 103, 135704.

Zeravcic, Z., Xu, N., Liu, A.J., Nagel, S.R., van Saarloos, W., 2009. Excitations of ellipsoid packings near jamming. EPL 87, 26001.

Zexin, Z., Ning, X., Chen, D.T.N., Yunker, P., Alsayed, A.M., Aptowicz, K.B., Habdas, P., Liu, A.J., Nagel, S.R., Yodh, A.G., 2009. Thermal vestige of the zero-temperature jamming transition. Nature 459, 230-233.

Zou, L.-N., Cheng, X., Rivers, M.L., Jaeger, H.M., Nagel, S.R., 2009. The packing of granular polymer chains. Science $326,408-410$. 